\title{
Hybrid Extragradient-Like Viscosity Methods for Generalized Mixed Equilibrium Problems, Variational Inclusions, and Optimization Problems
}

\author{
Lu-Chuan Ceng, ${ }^{1}$ Chi-Ming Chen, ${ }^{2}$ and Chin-Tzong Pang ${ }^{3}$ \\ ${ }^{1}$ Department of Mathematics, Shanghai Normal University, Scientific Computing Key Laboratory of Shanghai Universities, \\ Shanghai 200234, China \\ ${ }^{2}$ Department of Applied Mathematics, National Hsinchu University of Education, Hsinchu 30033, Taiwan \\ ${ }^{3}$ Department of Information Management, Yuan Ze University, Chung-Li 32003, Taiwan
}

Correspondence should be addressed to Chin-Tzong Pang; imctpang@saturn.yzu.edu.tw

Received 20 November 2013; Accepted 20 December 2013; Published 9 February 2014

Academic Editor: Erdal Karapinar

Copyright (C) 2014 Lu-Chuan Ceng et al. This is an open access article distributed under the Creative Commons Attribution License, which permits unrestricted use, distribution, and reproduction in any medium, provided the original work is properly cited.

\begin{abstract}
We introduce and analyze a new hybrid extragradient-like viscosity iterative algorithm for finding a common solution of a generalized mixed equilibrium problem, a finite family of variational inclusions for maximal monotone and inverse strongly monotone mappings, and a fixed point problem of infinitely many nonexpansive mappings in a real Hilbert space. Under some mild conditions, we prove the strong convergence of the sequence generated by the proposed algorithm to a common solution of these three problems which also solves an optimization problem.
\end{abstract}

\section{Introduction}

Let $H$ be a real Hilbert space with inner product $\langle\cdot, \cdot\rangle$ and norm $\|\cdot\|, C$ be a nonempty closed convex subset of $H$, and $P_{C}$ be the metric projection of $H$ onto $C$. Let $S: C \rightarrow H$ be a nonlinear mapping on $C$. We denote by $\operatorname{Fix}(S)$ the set of fixed points of $S$ and by $\mathbf{R}$ the set of all real numbers. A mapping $V$ is called strongly positive on $H$ if there exists a constant $\bar{\gamma} \in(0,1]$ such that

$$
\langle V x, x\rangle \geq \bar{\gamma}\|x\|^{2}, \quad \forall x \in H .
$$

A mapping $S: C \rightarrow H$ is called $L$-Lipschitz continuous if there exists a constant $L \geq 0$ such that

$$
\|S x-S y\| \leq L\|x-y\|, \quad \forall x, y \in C .
$$

In particular, if $L=1$ then $S$ is called a nonexpansive mapping; if $L \in[0,1)$ then $A$ is called a contraction.

Let $A: C \rightarrow H$ be a nonlinear mapping on $C$. Recall that the classical variational inequality problem (VIP) is to find a point $x \in C$ such that

$$
\langle A x, y-x\rangle \geq 0, \quad \forall y \in C .
$$

The solution set of VIP (3) is denoted by $\operatorname{VI}(C, A)$. The VIP (3) was first discussed by Lions [1] and has been extensively studied since then. See, for example, [2-5].

In 1976, Korpelevič [6] proposed an iterative algorithm for solving the VIP (3) in Euclidean space $\mathbf{R}^{n}$ :

$$
\begin{gathered}
y_{n}=P_{C}\left(x_{n}-\tau A x_{n}\right), \\
x_{n+1}=P_{C}\left(x_{n}-\tau A y_{n}\right), \quad \forall n \geq 0,
\end{gathered}
$$

with $\tau>0$ as a given number, which is known as the extragradient method. The literature on the VIP is vast and Korpelevich's extragradient method has received great attention given by many authors, see, for example, [7-23] and the references therein. Let $\varphi: C \rightarrow \mathbf{R}$ be a realvalued function, $A: H \rightarrow H$ be a nonlinear mapping, and $\Theta: C \times C \rightarrow \mathbf{R}$ be a bifunction. In 2008, Peng and Yao [14] introduced the following generalized mixed equilibrium problem (GMEP) of finding $x \in C$ such that

$$
\Theta(x, y)+\varphi(y)-\varphi(x)+\langle A x, y-x\rangle \geq 0, \quad \forall y \in C .
$$

We denote the set of solutions of GMEP (5) by $\operatorname{GMEP}(\Theta, \varphi, A)$. The GMEP (5) is very general in the 
sense that it includes, as special cases, optimization problems, variational inequalities, minimax problems, Nash equilibrium problems in noncooperative games, and others. The GMEP is further considered and studied; see, for example, [13, 16, 24-28].

We present some special cases of GMEP (5) as follows.

If $\varphi=0$, then GMEP (5) reduces to the generalized equilibrium problem (GEP) which is to find $x \in C$ such that

$$
\Theta(x, y)+\langle A x, y-x\rangle \geq 0, \quad \forall y \in C .
$$

This problem was introduced and studied by S. Takahashi and W. Takahashi [29]. The set of solutions of GEP is denoted by $\operatorname{GEP}(\Theta, A)$.

If $A=0$, then GMEP (5) reduces to the mixed equilibrium problem (MEP) which is to find $x \in C$ such that

$$
\Theta(x, y)+\varphi(y)-\varphi(x) \geq 0, \quad \forall y \in C .
$$

It is considered and studied in $[30,31]$. The set of solutions of MEP is denoted by $\operatorname{MEP}(\Theta, \varphi)$.

If $\varphi=0, A=0$, then GMEP (5) reduces to the equilibrium problem (EP) which is to find $x \in C$ such that

$$
\Theta(x, y) \geq 0, \quad \forall y \in C .
$$

This was considered and studied in [32, 33]. The set of solutions of EP is denoted by $\operatorname{EP}(\Theta)$. It is worth mentioning that the EP is a unified model of several problems, namely, variational inequality problems, optimization problems, saddle point problems, complementarity problems, fixed point problems, Nash equilibrium problems, and so forth.

For the bifunction $\Theta: C \times C \rightarrow \mathbf{R}$ and real-valued function $\varphi: C \rightarrow \mathbf{R}$ in the GMEP (5), as in [14], we assume that $\Theta$ is a bifunction satisfying conditions (H1)-(H4) and $\varphi$ is a lower semicontinuous and convex function with restriction (H5), where

(H1) $\Theta(x, x)=0$ for all $x \in C$;

(H2) $\Theta$ is monotone, that is; $\Theta(x, y)+\Theta(y, x) \leq 0$ for any $x, y \in C$;

$(\mathrm{H} 3) \Theta$ is upper-hemicontinuous, that is; for each $x, y, z \in$ C,

$$
\lim _{t \rightarrow 0^{+}} \sup _{t} \Theta(t z+(1-t) x, y) \leq \Theta(x, y) ;
$$

(H4) $\Theta(x, \cdot)$ is convex and lower semicontinuous for each $x \in C$;

(H5) for each $x \in H$ and $r>0$, there exists a bounded subset $D_{x} \subset C$ and $y_{x} \in C$ such that for any $z \in C \backslash D_{x}$,

$\Theta\left(z, y_{x}\right)+\varphi\left(y_{x}\right)-\varphi(z)+\frac{1}{r}\left\langle y_{x}-z, z-x\right\rangle<0$.

A differentiable function $K: H \rightarrow \mathbf{R}$ is called

(i) convex, if

$$
K(y)-K(x) \geq\left\langle K^{\prime}(x), y-x\right\rangle, \quad \forall x, y \in H,
$$

where $K^{\prime}$ is the Frechet derivative of $K$ at $x$; (ii) strongly convex, if there exists a constant $\sigma>0$ such that

$$
K(y)-K(x)-\left\langle K^{\prime}(x), y-x\right\rangle \geq \frac{\sigma}{2}\|x-y\|^{2}, \quad \forall x, y \in H .
$$

It is easy to see that if $K: H \rightarrow \mathbf{R}$ is a differentiable strongly convex function with constant $\sigma>0$, then $K^{\prime}: H \rightarrow$ $H$ is strongly monotone with constant $\sigma>0$.

Given a positive number $r>0$, let $S_{r}^{(\Theta, \varphi)}: H \rightarrow C$ be the solution set of the auxiliary mixed equilibrium problem; that is, for each $x \in H$,

$$
\begin{aligned}
S_{r}^{(\Theta, \varphi)}(x):=\{y \in C & : \Theta(y, z)+\varphi(z)-\varphi(y) \\
+ & \frac{1}{r}\left\langle K^{\prime}(y)-K^{\prime}(x), z-y\right\rangle \geq 0, \\
& \forall z \in C\} .
\end{aligned}
$$

In particular, whenever $K(x)=(1 / 2)\|x\|^{2}, \forall x \in H, S_{r}^{(\Theta, \varphi)}$ is rewritten as $T_{r}^{(\Theta, \varphi)}$.

Let $\Theta_{1}, \Theta_{2}: C \times C \rightarrow \mathbf{R}$ be two bifunctions and $B_{1}, B_{2}: C \rightarrow H$ be two nonlinear mappings. Consider the following system of generalized equilibrium problems (SGEP): find $\left(x^{*}, y^{*}\right) \in C \times C$ such that

$$
\begin{array}{r}
\Theta_{1}\left(x^{*}, x\right)+\left\langle B_{1} y^{*}, x-x^{*}\right\rangle+\frac{1}{\mu_{1}}\left\langle x^{*}-y^{*}, x-x^{*}\right\rangle \geq 0, \\
\forall x \in C, \\
\Theta_{2}\left(y^{*}, y\right)+\left\langle B_{2} x^{*}, y-y^{*}\right\rangle+\frac{1}{\mu_{2}}\left\langle y^{*}-x^{*}, y-y^{*}\right\rangle \geq 0, \\
\forall y \in C,
\end{array}
$$

where $\mu_{1}>0$ and $\mu_{2}>0$ are two constants. It is introduced and studied in [15], that the SGEP reduces to a system of variational inequalities whenever $\Theta_{1} \equiv \Theta_{2} \equiv 0$. It is worth mentioning that the system of variational inequalities is a tool to solve the Nash equilibrium problem for noncooperative games.

In 2010, Ceng and Yao [15] transformed the SGEP into a fixed point problem in the following way.

Proposition CY (see [15]). Let $\Theta_{1}, \Theta_{2}: C \times C \rightarrow \mathbf{R}$ be two bifunctions satisfying conditions $(\mathrm{H} 1)-(\mathrm{H} 4)$ and let $B_{i}: C \rightarrow$ $H$ be $\widetilde{\beta}_{i}$-inverse-strongly monotone for $i=1,2$. Let $\mu_{i} \in\left(0,2 \widetilde{\beta}_{i}\right)$ for $i=1,2$. Then, $\left(x^{*}, y^{*}\right) \in C \times C$ is a solution of SGEP if and only if $x^{*}$ is a fixed point of the mapping $G: C \rightarrow C$ defined by $G=T_{\mu_{1}}^{\Theta_{1}}\left(I-\mu_{1} B_{1}\right) T_{\mu_{2}}^{\Theta_{2}}\left(I-\mu_{2} B_{2}\right)$, where $y^{*}=T_{\mu_{2}}^{\Theta_{2}}\left(I-\mu_{2} B_{2}\right) x^{*}$. Here, one denotes the fixed point set of $G$ by $\operatorname{SGEP}(G)$.

Let $\left\{T_{n}\right\}_{n=1}^{\infty}$ be an infinite family of nonexpansive mappings on $H$ and $\left\{\lambda_{n}\right\}_{n=1}^{\infty}$ be a sequence of nonnegative 
numbers in $[0,1]$. For any $n \geq 1$, define a self-mapping $W_{n}$ on $H$ as follows:

$$
\begin{aligned}
& U_{n, n+1}=I, \\
& U_{n, n}=\lambda_{n} T_{n} U_{n, n+1}+\left(1-\lambda_{n}\right) I, \\
& U_{n, n-1}=\lambda_{n-1} T_{n-1} U_{n, n}+\left(1-\lambda_{n-1}\right) I, \\
& \vdots \\
& U_{n, k}=\lambda_{k} T_{k} U_{n, k+1}+\left(1-\lambda_{k}\right) I, \\
& U_{n, k-1}=\lambda_{k-1} T_{k-1} U_{n, k}+\left(1-\lambda_{k-1}\right) I, \\
& \vdots \\
& U_{n, 2}=\lambda_{2} T_{2} U_{n, 3}+\left(1-\lambda_{2}\right) I, \\
& W_{n}=U_{n, 1}=\lambda_{1} T_{1} U_{n, 2}+\left(1-\lambda_{1}\right) I .
\end{aligned}
$$

Such a mapping $W_{n}$ is called the $W$-mapping generated by $T_{n}, T_{n-1}, \ldots, T_{1}$ and $\lambda_{n}, \lambda_{n-1}, \ldots, \lambda_{1}$.

In 2011, for the case where $C=H$, let $f: H \rightarrow$ $H$ be a contraction, $K: H \rightarrow \mathrm{R}$ be differentiable and strongly convex, $\left\{\alpha_{n}\right\},\left\{\beta_{n}\right\} \subset(0,1)$ and $x_{0}, u \in H$ be given. Yao et al. [25] proposed the hybrid iterative algorithm for finding a common element of the set $\operatorname{MEP}(\Theta, \varphi)$ and the fixed set $\bigcap_{i=1}^{\infty} \operatorname{Fix}\left(T_{i}\right)$ of an infinite family of nonexpansive mappings $\left\{T_{i}\right\}_{i=1}^{\infty}$ on $H$ as follows:

$$
\begin{aligned}
\Theta & \left(y_{n}, z\right)+\varphi(z)-\varphi\left(y_{n}\right) \\
& +\frac{1}{r}\left\langle K^{\prime}\left(y_{n}\right)-K^{\prime}\left(x_{n}\right), z-y_{n}\right\rangle \geq 0, \quad z \in H, \\
x_{n+1}= & \alpha_{n}\left(u+\gamma f\left(x_{n}\right)\right)+\beta_{n} x_{n} \\
+ & \left(\left(1-\beta_{n}\right) I-\alpha_{n}(I+\mu V)\right) W_{n} y_{n}, \quad \forall n \geq 1 .
\end{aligned}
$$

They proved the strong convergence of the sequence generated by the hybrid iterative algorithm (16) to a point $x^{*} \epsilon$ $\bigcap_{i=1}^{\infty} \operatorname{Fix}\left(T_{i}\right) \cap \operatorname{MEP}(\Theta, \varphi)$ under some appropriate conditions. This point $x^{*}$ also solves the following optimization problem:

$$
\min _{x \in \bigcap_{n=1}^{\infty} \operatorname{Fix}\left(T_{n}\right) \cap \operatorname{MEP}(\Theta, \varphi)} \frac{\mu}{2}\langle V x, x\rangle+\frac{1}{2}\|x-u\|^{2}-h(x),
$$

where $h: H \rightarrow \mathbf{R}$ is the potential function of $\gamma f$.

Let $f: H \rightarrow H$ be a contraction and $V$ be a strongly positive bounded linear operator on $H$. Assume that $\varphi$ : $H \rightarrow \mathbf{R}$ is a lower semicontinuous and convex functional, that $\Theta, \Theta_{1}, \Theta_{2}: H \times H \rightarrow \mathbf{R}$ satisfy conditions (H1)-(H4), and that $A, B_{1}, B_{2}: H \rightarrow H$ are inverse-strongly monotone.
Very recently, Ceng et al. [16] introduced the following hybrid extragradient-like iterative algorithm:

$$
\begin{gathered}
z_{n}=S_{r_{n}}^{(\Theta, \varphi)}\left(x_{n}-r_{n} A x_{n}\right), \\
y_{n}=T_{\mu_{1}}^{\Theta_{1}}\left(I-\mu_{1} B_{1}\right) T_{\mu_{2}}^{\Theta_{2}}\left(I-\mu_{2} B_{2}\right) z_{n}, \\
x_{n+1}=\alpha_{n}\left(u+\gamma f\left(x_{n}\right)\right)+\beta_{n} x_{n} \\
+\left(\left(1-\beta_{n}\right) I-\alpha_{n}(I+\mu V)\right) W_{n} y_{n}, \quad \forall n \geq 0,
\end{gathered}
$$

for finding a common solution of GMEP (5), SGEP (14), and the fixed point problem of an infinite family of nonexpansive mappings $\left\{T_{i}\right\}_{i=1}^{\infty}$ on $H$, where $\left\{r_{n}\right\} \subset(0, \infty),\left\{\alpha_{n}\right\},\left\{\beta_{n}\right\} \subset$ $(0,1)$, and $x_{0}, u \in H$ are given. The authors proved the strong convergence of the sequence generated by the hybrid iterative algorithm (17) to a point $x^{*} \in \bigcap_{i=1}^{\infty} \operatorname{Fix}\left(T_{i}\right) \cap \operatorname{GMEP}(\Theta, \varphi, A) \cap$ $\operatorname{SGEP}(G)$ under some suitable conditions. This point $x^{*}$ also solves the following optimization problem:

$$
\begin{aligned}
& \underset{x \in \bigcap_{n=1}^{\infty} \operatorname{Fix}\left(T_{n}\right) \cap \min }{\min (\Theta, \varphi, A) \cap \operatorname{SGEP}(G)} \frac{\mu}{2}\langle V x, x\rangle \\
& +\frac{1}{2}\|x-u\|^{2}-h(x),
\end{aligned}
$$

where $h: H \rightarrow \mathbf{R}$ is the potential function of $\gamma f$.

On the other hand, let $B$ be a single-valued mapping of $C$ into $H$ and $R$ be a set-valued mapping with $D(R)=C$. Consider the following variational inclusion: find a point $x \in$ $C$ such that

$$
0 \in B x+R x
$$

We denote by $I(B, R)$ the solution set of the variational inclusion (18). In particular, if $B=R=0$, then $I(B, R)=C$. If $B=0$, then problem (18) becomes the inclusion problem introduced by Rockafellar [34]. It is known that problem (18) provides a convenient framework for the unified study of optimal solutions in many optimization related areas including mathematical programming, complementarity problems, variational inequalities, optimal control, mathematical economics, equilibria and game theory, etc. Let a set-valued mapping $R: D(R) \subset H \rightarrow 2^{H}$ be maximal monotone. We define the resolvent operator $J_{R, \lambda}: H \rightarrow \overline{D(R)}$ associated with $R$ and $\lambda$ as follows:

$$
J_{R, \lambda}=(I+\lambda R)^{-1}, \quad \forall x \in H,
$$

where $\lambda$ is a positive number.

In 1998, Huang [35] studied problem (18) in the case where $R$ is maximal monotone and $B$ is strongly monotone and Lipschitz continuous with $D(R)=C=H$. Subsequently, Zeng et al. [36] further studied problem (18) in the case which is more general than Huang's [35]. Moreover, the authors [36] obtained the same strong convergence conclusion as in Huang's result [35]. In addition, the authors also gave the geometric convergence rate estimate for approximate solutions. Also, various types of iterative algorithms for solving variational inclusions have been further studied and 
developed; for more details, refer to [21, 26, 37, 38] and the references therein.

Motivated and inspired by the above facts, we, in this paper, introduce and analyze a new iterative algorithm by a hybrid extragradient-like viscosity method for finding a common element of the set of solutions of a generalized mixed equilibrium problem, the set of solutions of a finite family of variational inclusions for maximal monotone and inverse strong monotone mappings, and the set of fixed points of a countable family of nonexpansive mappings in a real Hilbert space. Under some appropriate conditions, we prove the strong convergence of the sequence generated by the proposed algorithm to a common solution of these three problems. Such a solution also solves an optimization problem. Several special cases are also discussed. The results presented in this paper are the supplement, extension, improvement, and generalization of the previously known results in this area.

\section{Preliminaries}

Throughout this paper, we assume that $H$ is a real Hilbert space whose inner product and norm are denoted by $\langle\cdot, \cdot\rangle$ and $\|\cdot\|$, respectively. Let $C$ be a nonempty closed convex subset of $H$. We write $x_{n} \rightarrow x$ to indicate that the sequence $\left\{x_{n}\right\}$ converges weakly to $x$ and $x_{n} \rightarrow x$ to indicate that the sequence $\left\{x_{n}\right\}$ converges strongly to $x$. Moreover, we use $\omega_{w}\left(x_{n}\right)$ to denote the weak $\omega$-limit set of the sequence $\left\{x_{n}\right\}$; that is,

$$
\begin{aligned}
\omega_{w}\left(x_{n}\right):= & \{x \in H: \\
& \left.x_{n_{i}} \rightarrow x \text { for some subsequence }\left\{x_{n_{i}}\right\} \text { of }\left\{x_{n}\right\}\right\} .
\end{aligned}
$$

Recall that a mapping $A: C \rightarrow H$ is called

(i) monotone if

$$
\langle A x-A y, x-y\rangle \geq 0, \quad \forall x, y \in C ;
$$

(ii) $\eta$-strongly monotone if there exists a constant $\eta>0$ such that

$$
\langle A x-A y, x-y\rangle \geq \eta\|x-y\|^{2}, \quad \forall x, y \in C ;
$$

(iii) $\zeta$-inverse-strongly monotone if there exists a constant $\zeta>0$ such that

$$
\langle A x-A y, x-y\rangle \geq \zeta\|A x-A y\|^{2}, \quad \forall x, y \in C .
$$

It is easy to see that the projection $P_{C}$ is 1 -ism. Inverse strongly monotone (also referred to as cocoercive) operators have been applied widely in solving practical problems in various fields.

The metric (or nearest point) projection from $H$ onto $C$ is the mapping $P_{C}: H \rightarrow C$ which assigns to each point $x \in H$ the unique point $P_{C} x \in C$ satisfying the property

$$
\left\|x-P_{C} x\right\|=\inf _{y \in C}\|x-y\|=: d(x, C) .
$$

Some important properties of projections are gathered in the following proposition.

Proposition 1. For given $x \in H$ and $z \in C$ :

(i) $z=P_{C} x \Leftrightarrow\langle x-z, y-z\rangle \leq 0, \forall y \in C$;

(ii) $z=P_{C} x \Leftrightarrow\|x-z\|^{2} \leq\|x-y\|^{2}-\|y-z\|^{2}, \forall y \in C$;

(iii) $\left\langle P_{C} x-P_{C} y, x-y\right\rangle \geq\left\|P_{C} x-P_{C} y\right\|^{2}, \forall y \in H$. (This implies that $P_{C}$ is nonexpansive and monotone.)

By using the technique of [31], we can readily obtain the following elementary result.

Proposition 2 (see [16, Lemma 1 and Proposition 1]). Let $C$ be a nonempty closed convex subset of a real Hilbert space $H$ and let $\varphi: C \rightarrow \mathbf{R}$ be a lower semicontinuous and convex function. Let $\Theta: C \times C \rightarrow \mathbf{R}$ be a bifunction satisfying the conditions (H1)-(H4). Assume that

(i) $\mathrm{K}: H \rightarrow \mathbf{R}$ is strongly convex with constant $\sigma>0$ and the function $x \mapsto\left\langle y-x, K^{\prime}(x)\right\rangle$ is weakly upper semicontinuous for each $y \in H$;

(ii) for each $x \in H$ and $r>0$, there exists a bounded subset $D_{x} \subset C$ and $y_{x} \in C$ such that for any $z \in C \backslash D_{x}$,

$\Theta\left(z, y_{x}\right)+\varphi\left(y_{x}\right)-\varphi(z)+\frac{1}{r}\left\langle K^{\prime}(z)-K^{\prime}(x), y_{x}-z\right\rangle<0$.

Then the following hold:

(a) for each $x \in H, S_{r}^{(\Theta, \varphi)}(x) \neq \emptyset$;

(b) $S_{r}^{(\Theta, \varphi)}$ is single-valued;

(c) $S_{r}^{(\Theta, \varphi)}$ is nonexpansive if $K^{\prime}$ is Lipschitz continuous with constant $v>0$ and

$$
\begin{aligned}
& \left\langle K^{\prime}\left(x_{1}\right)-K^{\prime}\left(x_{2}\right), u_{1}-u_{2}\right\rangle \\
& \quad \geq\left\langle K^{\prime}\left(u_{1}\right)-K^{\prime}\left(u_{2}\right), u_{1}-u_{2}\right\rangle, \quad \forall\left(x_{1}, x_{2}\right) \in H \times H,
\end{aligned}
$$

where $u_{i}=S_{r}^{(\Theta, \varphi)}\left(x_{i}\right)$ for $i=1,2$;

(d) for all $s, t>0$ and $x \in H$

$$
\begin{aligned}
& \left\langle K^{\prime}\left(S_{s}^{(\Theta, \varphi)} x\right)-K^{\prime}\left(S_{t}^{(\Theta, \varphi)} x\right), S_{s}^{(\Theta, \varphi)} x-S_{t}^{(\Theta, \varphi)} x\right\rangle \\
& \leq \frac{s-t}{s}\left\langle K^{\prime}\left(S_{s}^{(\Theta, \varphi)} x\right)-K^{\prime}(x), S_{s}^{(\Theta, \varphi)} x-S_{t}^{(\Theta, \varphi)} x\right\rangle ;
\end{aligned}
$$

(e) $\operatorname{Fix}\left(S_{r}^{(\Theta, \varphi)}\right)=\operatorname{MEP}(\Theta, \varphi)$;

(f) $\operatorname{MEP}(\Theta, \varphi)$ is closed and convex.

In particular, whenever $\Theta: C \times C \rightarrow \mathbf{R}$ is a bifunction satisfying the conditions $(\mathrm{H} 1)-(\mathrm{H} 4)$ and $K(x)=(1 / 2)\|x\|^{2}, \forall x \in H$, then, that is, for any $x, y \in H$,

$$
\left\|S_{r}^{(\Theta, \varphi)} x-S_{r}^{(\Theta, \varphi)} y\right\|^{2} \leq\left\langle S_{r}^{(\Theta, \varphi)} x-S_{r}^{(\Theta, \varphi)} y, x-y\right\rangle
$$


$\left(S_{r}^{(\Theta, \varphi)}\right.$ is firmly nonexpansive $)$ and

$$
\begin{array}{r}
\left\|S_{s}^{(\Theta, \varphi)} x-S_{t}^{(\Theta, \varphi)} x\right\| \leq \frac{|s-t|}{s}\left\|S_{s}^{(\Theta, \varphi)} x-x\right\|, \\
\forall s, t>0, x \in H .
\end{array}
$$

In this case, $S_{r}^{(\Theta, \varphi)}$ is rewritten as $T_{r}^{(\Theta, \varphi)}$. If, in addition, $\varphi \equiv 0$, then $T_{r}^{(\Theta, \varphi)}$ is rewritten as $T_{r}^{\Theta}$ (see [15, Lemma 2.1] for more details).

Remark 3. Suppose $K: H \rightarrow \mathbf{R}$ is strongly convex with constant $\sigma>0$ and $K^{\prime}: H \rightarrow H$ is Lipschitz continuous with constant $\nu>0$. Then $K^{\prime}: H \rightarrow H$ is $\sigma$-strongly monotone and $\nu$-Lipschitz continuous with positive constants $\sigma, \nu>0$. Utilizing Proposition 2(d) we obtain that for all $s, t>0$ and $x \in H$

$$
\begin{aligned}
\sigma \| S_{s}^{(\Theta, \varphi)} & x-S_{t}^{(\Theta, \varphi)} x \|^{2} \\
& \leq\left\langle K^{\prime}\left(S_{s}^{(\Theta, \varphi)} x\right)-K^{\prime}\left(S_{t}^{(\Theta, \varphi)} x\right), S_{s}^{(\Theta, \varphi)} x-S_{t}^{(\Theta, \varphi)} x\right\rangle \\
& \leq \frac{s-t}{s}\left\langle K^{\prime}\left(S_{s}^{(\Theta, \varphi)} x\right)-K^{\prime}(x), S_{s}^{(\Theta, \varphi)} x-S_{t}^{(\Theta, \varphi)} x\right\rangle \\
& \leq \frac{|s-t|}{s}\left\|K^{\prime}\left(S_{s}^{(\Theta, \varphi)} x\right)-K^{\prime}(x)\right\|\left\|S_{s}^{(\Theta, \varphi)} x-S_{t}^{(\Theta, \varphi)} x\right\| \\
& \leq \frac{|s-t|}{s} \nu\left\|S_{s}^{(\Theta, \varphi)} x-x\right\|\left\|S_{s}^{(\Theta, \varphi)} x-S_{t}^{(\Theta, \varphi)} x\right\|,
\end{aligned}
$$

which immediately implies that

$$
\left\|S_{s}^{(\Theta, \varphi)} x-S_{t}^{(\Theta, \varphi)} x\right\| \leq \frac{|s-t|}{s} \cdot \frac{\nu}{\sigma}\left\|S_{s}^{(\Theta, \varphi)} x-x\right\| .
$$

We need some facts and tools in a real Hilbert space $H$ which are listed as lemmas below.

Lemma 4. Let $X$ be a real inner product space. Then there holds the following inequality:

$$
\|x+y\|^{2} \leq\|x\|^{2}+2\langle y, x+y\rangle, \quad \forall x, y \in X .
$$

Lemma 5. Let $H$ be a real Hilbert space. Then the following hold:

(a) $\|x-y\|^{2}=\|x\|^{2}-\|y\|^{2}-2\langle x-y, y\rangle$ for all $x, y \in H$;

(b) $\|\lambda x+\mu y\|^{2}=\lambda\|x\|^{2}+\mu\|y\|^{2}-\lambda \mu\|x-y\|^{2}$ for all $x, y \in$ $H$ and $\lambda, \mu \in[0,1]$ with $\lambda+\mu=1$;

(c) if $\left\{x_{n}\right\}$ is a sequence in $H$ such that $x_{n} \rightarrow x$, it follows that

$$
\begin{array}{r}
\limsup _{n \rightarrow \infty}\left\|x_{n}-y\right\|^{2}=\lim _{n \rightarrow \infty} \sup \left\|x_{n}-x\right\|^{2}+\|x-y\|^{2}, \\
\forall y \in H .
\end{array}
$$

We have the following crucial lemmas concerning the $W$ mappings defined by (15).
Lemma 6 (see [39, Lemma 3.2]). Let $\left\{T_{n}\right\}_{n=1}^{\infty}$ be a sequence of nonexpansive self-mappings on $H$ such that $\bigcap_{n=1}^{\infty} \operatorname{Fix}\left(T_{n}\right) \neq \emptyset$ and let $\left\{\lambda_{n}\right\}$ be a sequence in $(0, b]$ for some $b \in(0,1)$. Then, for every $x \in H$ and $k \geq 1$ the limit $\lim _{n \rightarrow \infty} U_{n, k} x$ exists, where $U_{n, k}$ is defined by (15).

Remark 7 (see [40, Remark 3.1]). It can be known from Lemma 6 that if $D$ is a nonempty bounded subset of $H$, then for $\epsilon>0$ there exists $n_{0} \geq k$ such that for all $n>n_{0}$

$$
\sup _{x \in D}\left\|U_{n, k} x-U_{k} x\right\| \leq \epsilon .
$$

Remark 8 (see [40, Remark 3.2]). Utilizing Lemma 6, we define a mapping $W: H \rightarrow H$ as follows:

$$
W x=\lim _{n \rightarrow \infty} W_{n} x=\lim _{n \rightarrow \infty} U_{n, 1} x, \quad \forall x \in H .
$$

Such a $W$ is called the $W$-mapping generated by $T_{1}, T_{2}, \ldots$ and $\lambda_{1}, \lambda_{2}, \ldots$. Since $W_{n}$ is nonexpansive, $W: H \rightarrow H$ is also nonexpansive. Indeed, observe that for each $x, y \in H$

$$
\|W x-W y\|=\lim _{n \rightarrow \infty}\left\|W_{n} x-W_{n} y\right\| \leq\|x-y\| .
$$

If $\left\{x_{n}\right\}$ is a bounded sequence in $H$, then we put $D=\left\{x_{n}\right.$ : $n \geq 1\}$. Hence, it is clear from Remark 3 that for an arbitrary $\epsilon>0$ there exists $N_{0} \geq 1$ such that for all $n>N_{0}$

$$
\begin{aligned}
& \left\|W_{n} x_{n}-W x_{n}\right\| \\
& \quad=\left\|U_{n, 1} x_{n}-U_{1} x_{n}\right\| \leq \sup _{x \in D}\left\|U_{n, 1} x-U_{1} x\right\| \leq \epsilon .
\end{aligned}
$$

This implies that

$$
\lim _{n \rightarrow \infty}\left\|W_{n} x_{n}-W x_{n}\right\|=0
$$

Lemma 9 (see [39, Lemma 3.3]). Let $\left\{T_{n}\right\}_{n=1}^{\infty}$ be a sequence of nonexpansive self-mappings on $H$ such that $\bigcap_{n=1}^{\infty} \operatorname{Fix}\left(T_{n}\right) \neq \emptyset$ and let $\left\{\lambda_{n}\right\}$ be a sequence in $(0, b]$ for some $b \in(0,1)$. Then, $\operatorname{Fix}(W)=\bigcap_{n=1}^{\infty} \operatorname{Fix}\left(T_{n}\right)$.

Lemma 10 (see [41, Theorem 10.4 (Demiclosedness Principle)]). Let $C$ be a nonempty closed convex subset of a real Hilbert space $H$. Let $T: C \rightarrow C$ be nonexpansive. Then $I-T$ is demiclosed on $C$. That is, whenever $\left\{x_{n}\right\}$ is a sequence in $C$ weakly converging to some $x \in C$ and the sequence $\left\{(I-T) x_{n}\right\}$ strongly converges to some $y$, it follows that $(I-T) x=y$. Here $I$ is the identity operator of $H$.

Lemma 11. Let $A: C \rightarrow H$ be a monotone mapping. In the context of the variational inequality problem the characterization of the projection (see Proposition 1(i)) implies

$$
u \in V I(C, A) \Longleftrightarrow u=P_{C}(u-\lambda A u), \quad \forall \lambda>0 .
$$

The following lemma can be easily proven, and therefore, we omit the proof.

Lemma 12. Let $f: H \rightarrow H$ be an l-Lipschitzian mapping with constant $l \geq 0$ and $F: H \rightarrow H$ be $a$-Lipschitzian and 
$\eta$-strongly monotone operator with positive constants $\kappa, \eta>0$. Then for $0 \leq \gamma l<\mu \eta$,

$$
\begin{array}{r}
\langle(\mu F-\gamma f) x-(\mu F-\gamma f) y, x-y\rangle \geq(\mu \eta-\gamma l)\|x-y\|^{2}, \\
\forall x, y \in H .
\end{array}
$$

That is, $\mu F-\gamma f$ is strongly monotone with constant $\mu \eta-\gamma l$.

Let $C$ be a nonempty closed convex subset of a real Hilbert space $H$. We introduce some notations. Let $\lambda$ be a number in $(0,1]$ and let $\mu>0$. Associating with a nonexpansive mapping $T: C \rightarrow C$, we define the mapping $T^{\lambda}: C \rightarrow H$ by

$$
T^{\lambda} x:=T x-\lambda \mu F(T x), \quad \forall x \in C,
$$

where $F: H \rightarrow H$ is an operator such that, for some positive constants $\kappa, \eta>0, F$ is $\kappa$-Lipschitzian and $\eta$-strongly monotone on $H$; that is, $F$ satisfies the conditions:

$$
\begin{gathered}
\|F x-F y\| \leq \kappa\|x-y\|, \\
\langle F x-F y, x-y\rangle \geq \eta\|x-y\|^{2}
\end{gathered}
$$

for all $x, y \in H$.

Lemma 13 (see [42, Lemma 3.1]). $T^{\lambda}$ is a contraction provided $0<\mu<\left(2 \eta / \kappa^{2}\right)$; that is,

$$
\left\|T^{\lambda} x-T^{\lambda} y\right\| \leq(1-\lambda \tau)\|x-y\|, \quad \forall x, y \in C,
$$

where $\tau=1-\sqrt{1-\mu\left(2 \eta-\mu \kappa^{2}\right)} \in(0,1]$.

Recall that a set-valued mapping $T: D(T) \subset H \rightarrow 2^{H}$ is called monotone if for all $x, y \in D(T), f \in T x$, and $g \in T y$ imply

$$
\langle f-g, x-y\rangle \geq 0 .
$$

A set-valued mapping $T$ is called maximal monotone if $T$ is monotone and $(I+\lambda T) D(T)=H$ for each $\lambda>0$, where $I$ is the identity mapping of $H$. We denote by $G(T)$ the graph of $T$. It is known that a monotone mapping $T$ is maximal if and only if, for $(x, f) \in H \times H,\langle f-g, x-y\rangle \geq 0$ for every $(y, g) \in G(T)$ implies that $f \in T x$. Next we provide an example to illustrate the concept of maximal monotone mapping.

Let $A: C \rightarrow H$ be a monotone, $k$-Lipschitz-continuous mapping and let $N_{C} v$ be the normal cone to $C$ at $v \in C$; that is,

$$
N_{C} v=\{w \in H:\langle v-u, w\rangle \geq 0, \forall u \in C\} .
$$

Define

$$
T v= \begin{cases}A v+N_{C} v, & \text { if } v \in C, \\ \emptyset, & \text { if } v \notin C .\end{cases}
$$

Then, $T$ is maximal monotone and $0 \in T v$ if and only if $v \in$ $\mathrm{VI}(C, A)$; see [34].

Assume that $R: D(R) \subset H \rightarrow 2^{H}$ is a maximal monotone mapping. Let $\lambda>0$. In terms of Huang [35] (see also [36]), there holds the following property for the resolvent operator $J_{R, \lambda}: H \rightarrow \overline{D(R)}$.
Lemma 14. $J_{R, \lambda}$ is single-valued and firmly nonexpansive; that is,

$$
\left\langle J_{R, \lambda} x-J_{R, \lambda} y, x-y\right\rangle \geq\left\|J_{R, \lambda} x-J_{R, \lambda} y\right\|^{2}, \quad \forall x, y \in H .
$$

Consequently, $J_{R, \lambda}$ is nonexpansive and monotone.

Lemma 15 (see [21]). Let $R$ be a maximal monotone mapping with $D(R)=C$. Then for any given $\lambda>0, u \in C$ is a solution of problem (17) if and only if $u \in C$ satisfies

$$
u=J_{R, \lambda}(u-\lambda B u)
$$

Lemma 16 (see [36]). Let $R$ be a maximal monotone mapping with $D(R)=C$ and let $B: C \rightarrow H$ be a strongly monotone, continuous, and single-valued mapping. Then for each $z \in H$, the equation $z \in(B+\lambda R) x$ has a unique solution $x_{\lambda}$ for $\lambda>0$.

Lemma 17 (see [21]). Let $R$ be a maximal monotone mapping with $D(R)=C$ and $B: C \rightarrow H$ be a monotone, continuous, and single-valued mapping. Then $(I+\lambda(R+B)) C=H$ for each $\lambda>0$. In this case, $R+B$ is maximal monotone.

Lemma 18 (see [30]). Let $C$ be a nonempty closed convex subset of a real Hilbert space $H$, and $g: C \rightarrow \mathbf{R} \cup+\infty$ be a proper lower semicontinuous differentiable convex function. If $x^{*}$ is a solution the minimization problem

$$
g\left(x^{*}\right)=\inf _{x \in C} g(x)
$$

then,

$$
\left\langle g^{\prime}(x), x-x^{*}\right\rangle \geq 0, \quad \forall x \in C
$$

In particular, if $x^{*}$ solves $(O P)$, then

$$
\left\langle u+(\gamma f-(I+\mu V)) x^{*}, x-x^{*}\right\rangle \leq 0 .
$$

Lemma 19 (see [43]). Let $\left\{s_{n}\right\}$ be a sequence of nonnegative real numbers satisfying

$$
s_{n+1} \leq\left(1-\alpha_{n}\right) s_{n}+\alpha_{n} \beta_{n}+\gamma_{n}, \quad \forall n \geq 1,
$$

where $\left\{\alpha_{n}\right\},\left\{\beta_{n}\right\}$, and $\left\{\gamma_{n}\right\}$ are three real sequences satisfying the conditions:

(i) $\left\{\alpha_{n}\right\} \subset[0,1], \sum_{n=1}^{\infty} \alpha_{n}=\infty$;

(ii) $\limsup _{n \rightarrow \infty} \beta_{n} \leq 0$;

(iii) $\gamma_{n} \geq 0(\forall n \geq 1), \sum_{n=1}^{\infty} \gamma_{n}<\infty$.

Then $\lim _{n \rightarrow \infty} s_{n}=0$.

\section{Main Results}

We introduce and analyze a new iterative algorithm by hybrid extragradient-like viscosity method for finding a common element of the set of solutions of a generalized mixed equilibrium problem, the set of solutions of a finite family of variational inclusions for maximal monotone and inverse 
strong monotone mappings, and the set of fixed points of a countable family of nonexpansive mappings in a real Hilbert space. Under appropriate conditions imposed on the parameter sequences we will prove a strong convergence of the proposed algorithm.

Theorem 20. Let $C$ be a nonempty closed convex subset of a real Hilbert space $H$. Let $N$ be an integer. Let $\Theta$ be a bifunction from $\mathrm{C} \times \mathrm{C}$ to $\mathbf{R}$ satisfying $(\mathrm{H} 1)-(\mathrm{H} 4)$ and $\varphi: C \rightarrow \mathbf{R}$ be a lower semicontinuous and convex functional. Let $R_{i}: C \rightarrow 2^{H}$ be a maximal monotone mapping and let $A: H \rightarrow H$ and $B_{i}: C \rightarrow H$ be $\zeta$-inverse strongly monotone and $\eta_{i}{ }^{-}$ inverse strongly monotone, respectively, where $i \in\{1,2, \ldots, N\}$. Let $\left\{T_{n}\right\}_{n=1}^{\infty}$ be a sequence of nonexpansive self-mappings on $H$ and $\left\{\lambda_{n}\right\}$ be a sequence in $(0, b]$ for some $b \in(0,1)$. Let $F: H \rightarrow H$ be a $\kappa$-Lipschitzian and $\eta$-strongly monotone operator with positive constants $\kappa, \eta>0$. Let $0<\mu<$ $\left(2 \eta / \kappa^{2}\right)$ and $\tau=1-\sqrt{1-\mu\left(2 \eta-\mu \kappa^{2}\right)}$. Let $f: H \rightarrow H$ be an l-Lipschitzian mapping with $0 \leq \gamma l<\tau$. Let $W_{n}$ be the $W$-mapping defined by (15) and $V$ be a $\bar{\gamma}$-strongly positive bounded linear operator with $\gamma l<(1+\mu) \bar{\gamma}$. Assume that $\Omega:=\bigcap_{n=1}^{\infty} \operatorname{Fix}\left(T_{n}\right) \cap \operatorname{GMEP}(\Theta, \varphi, A) \cap \bigcap_{i=1}^{N} I\left(B_{i}, R_{i}\right)$ is nonempty. Suppose $\left\{\alpha_{n}\right\},\left\{\beta_{n}\right\}$, and $\left\{\sigma_{n}\right\}$ are three sequences in $(0,1)$. Assume that

(i) $\mathrm{K}: H \rightarrow \mathbf{R}$ is strongly convex with constant $\sigma>0$ and its derivative $K^{\prime}$ is Lipschitz continuous with constant $v>0$ such that the function $x \mapsto\left\langle y-x, K^{\prime}(x)\right\rangle$ is weakly upper semicontinuous for each $y \in H$;

(ii) for each $x \in H$, there exist a bounded subset $D_{x} \subset C$ and $z_{x} \in C$ such that for any $y \notin D_{x}$,

$$
\begin{aligned}
& \Theta\left(y, z_{x}\right)+\varphi\left(z_{x}\right)-\varphi(y) \\
& +\frac{1}{r}\left\langle K^{\prime}(y)-K^{\prime}(x), z_{x}-y\right\rangle<0 ;
\end{aligned}
$$

(iii) $\lim _{n \rightarrow \infty} \sigma_{n}=\lim _{n \rightarrow \infty}\left(\alpha_{n} / \sigma_{n}\right)=0$, limsup $\sup _{n \rightarrow \infty}$ $\left(\alpha_{n+1} / \alpha_{n}\right)<\infty, \sum_{n=1}^{\infty} \sigma_{n}=\infty$ and

$$
0<\liminf _{n \rightarrow \infty} \beta_{n} \leq \lim _{n \rightarrow \infty} \sup _{n} \beta_{n}<1
$$

(iv) $\mu_{i} \in\left(0,2 \eta_{i}\right), i \in\{1,2, \ldots, N\}$, and $\left\{r_{n}\right\} \subset[0,2 \zeta]$ satisfies

$$
0<\liminf _{n \rightarrow \infty} r_{n} \leq \limsup _{n \rightarrow \infty} r_{n}<2 \zeta
$$

(v) $\sum_{n=1}^{\infty}\left(\left|\beta_{n+1}-\beta_{n}\right|+\left|\sigma_{n+1}-\sigma_{n}\right|+\left|r_{n+1}-r_{n}\right|\right)<\infty$.
Given $x_{1} \in H$ arbitrarily, then the sequence $\left\{x_{n}\right\}$ generated iteratively by

$$
\begin{gathered}
u_{n}=S_{r_{n}}^{(\Theta, \varphi)}\left(I-r_{n} A\right) x_{n}, \\
z_{n}=J_{R_{N}, \mu_{N}}\left(I-\mu_{N} B_{N}\right) J_{R_{N-1}, \mu_{N-1}}\left(I-\mu_{N-1} B_{N-1}\right) \\
\cdots J_{R_{1}, \mu_{1}}\left(I-\mu_{1} B_{1}\right) u_{n}, \\
y_{n}=\alpha_{n}\left(u+\gamma f\left(x_{n}\right)\right)+\beta_{n} x_{n} \\
+\left(\left(1-\beta_{n}\right) I-\alpha_{n}(I+\mu V)\right) W_{n} z_{n}, \\
x_{n+1}=\sigma_{n} \gamma f\left(y_{n}\right)+\left(I-\sigma_{n} \mu F\right) W_{n} y_{n}, \\
\forall n \geq 1,
\end{gathered}
$$

converges strongly to $x^{*} \in \Omega$ provided $S_{r}^{(\Theta, \varphi)}$ is firmly nonexpansive, where $x^{*}=P_{\Omega}(I-(\mu F-\gamma f)) x^{*}$ is a unique solution of the VIP:

$$
\left\langle(\gamma f-\mu F) x^{*}, y-x^{*}\right\rangle \leq 0, \quad \forall y \in \Omega
$$

Proof. Since $\lim _{n \rightarrow \infty} \alpha_{n}=0$ and $0<\liminf _{n \rightarrow \infty} \beta_{n} \leq$ $\lim \sup _{n \rightarrow \infty} \beta_{n}<1$, we may assume, without loss of generality, that $\alpha_{n} \leq\left(1-\beta_{n}\right)(1+\mu\|V\|)^{-1}$. Since $V$ is a $\bar{\gamma}$ strongly positive bounded linear operator on $H$, we know that

$$
\|V\|=\sup \{\langle V u, u\rangle: u \in H,\|u\|=1\}
$$

Observe that

$$
\begin{aligned}
\left\langle\left(\left(1-\beta_{n}\right) I-\alpha_{n}(I+\mu V)\right) u, u\right\rangle & =1-\beta_{n}-\alpha_{n}-\alpha_{n} \mu\langle V u, u\rangle \\
& \geq 1-\beta_{n}-\alpha_{n}-\alpha_{n} \mu\|V\| \\
& \geq 0 ;
\end{aligned}
$$

that is, $\left(1-\beta_{n}\right) I-\alpha_{n}(I+\mu V)$ is positive. It follows that

$$
\begin{aligned}
& \left\|\left(1-\beta_{n}\right) I-\alpha_{n}(I+\mu V)\right\| \\
& \quad=\sup \left\{\left\langle\left(\left(1-\beta_{n}\right) I-\alpha_{n}(I+\mu V)\right) u, u\right\rangle: u \in H,\|u\|=1\right\} \\
& \quad=\sup \left\{1-\beta_{n}-\alpha_{n}-\alpha_{n} \mu\langle V u, u\rangle: u \in H,\|u\|=1\right\} \\
& \quad \leq 1-\beta_{n}-\alpha_{n}-\alpha_{n} \mu \bar{\gamma} .
\end{aligned}
$$

Put

$$
\begin{aligned}
\Lambda^{i}= & J_{R_{i}, \mu_{i}}\left(I-\mu_{i} B_{i}\right) J_{R_{i-1}, \mu_{i-1}}\left(I-\mu_{i-1} B_{i-1}\right) \\
& \quad \ldots J_{R_{1}, \mu_{1}}\left(I-\mu_{1} B_{1}\right)
\end{aligned}
$$

for all $i \in\{1,2, \ldots, N\}$ and $\Lambda^{0}=I$, where $I$ is the identity mapping on $H$. Then we have that $z_{n}=\Lambda^{N} u_{n}$. 
We divide the rest of the proof into several steps.

Step 1. We show that $\left\{x_{n}\right\}$ is bounded. Indeed, take $p \in \Omega$ arbitrarily. Since $p=S_{r_{n}}^{(\Theta, \varphi)}\left(p-r_{n} A p\right), A$ is $\zeta$-inverse strongly monotone and $0 \leq r_{n} \leq 2 \zeta$, we have, for any $n \geq 1$,

$$
\begin{aligned}
& \left\|u_{n}-p\right\|^{2} \\
& =\left\|S_{r_{n}}^{(\Theta, \varphi)}\left(I-r_{n} A\right) x_{n}-S_{r_{n}}^{(\Theta, \varphi)}\left(I-r_{n} A\right) p\right\|^{2} \\
& \leq\left\|\left(I-r_{n} A\right) x_{n}-\left(I-r_{n} A\right) p\right\|^{2} \\
& =\left\|\left(x_{n}-p\right)-r_{n}\left(A x_{n}-A p\right)\right\|^{2} \\
& =\left\|x_{n}-p\right\|^{2}-2 r_{n}\left\langle x_{n}-p, A x_{n}-A p\right\rangle+r_{n}^{2}\left\|A x_{n}-A p\right\|^{2} \\
& \leq\left\|x_{n}-p\right\|^{2}-2 r_{n} \zeta\left\|A x_{n}-A p\right\|^{2}+r_{n}^{2}\left\|A x_{n}-A p\right\|^{2} \\
& =\left\|x_{n}-p\right\|^{2}+r_{n}\left(r_{n}-2 \zeta\right)\left\|A x_{n}-A p\right\|^{2} \\
& \leq\left\|x_{n}-p\right\|^{2} .
\end{aligned}
$$

Since $p=J_{R_{i}, \mu_{i}}\left(I-\mu_{i} B_{i}\right) p, \Lambda^{i} p=p$, and $B_{i}$ is $\eta_{i}$-inverse strongly monotone, where $\mu_{i} \in\left(0,2 \eta_{i}\right), i \in\{1,2, \ldots, N\}$, by Lemma 14 we deduce that for each $n \geq 1$

$\left\|z_{n}-p\right\|^{2}$

$=\left\|J_{R_{N}, \mu_{N}}\left(I-\mu_{N} B_{N}\right) \Lambda^{N-1} u_{n}-J_{R_{N}, \mu_{N}}\left(I-\mu_{N} B_{N}\right) \Lambda^{N-1} p\right\|^{2}$

$\leq\left\|\left(I-\mu_{N} B_{N}\right) \Lambda^{N-1} u_{n}-\left(I-\mu_{N} B_{N}\right) \Lambda^{N-1} p\right\|^{2}$

$=\left\|\left(\Lambda^{N-1} u_{n}-\Lambda^{N-1} p\right)-\mu_{N}\left(B_{N} \Lambda^{N-1} u_{n}-B_{N} \Lambda^{N-1} p\right)\right\|^{2}$

$\leq\left\|\Lambda^{N-1} u_{n}-\Lambda^{N-1} p\right\|^{2}+\mu_{N}\left(\mu_{N}-2 \eta_{N}\right)$

$\times\left\|B_{N} \Lambda^{N-1} u_{n}-B_{N} \Lambda^{N-1} p\right\|^{2}$

$\leq\left\|\Lambda^{N-1} u_{n}-\Lambda^{N-1} p\right\|^{2}$

$\leq\left\|\Lambda^{0} u_{n}-\Lambda^{0} p\right\|^{2}$

$=\left\|u_{n}-p\right\|^{2}$.

Combining (62) and (63), we have

$$
\left\|z_{n}-p\right\| \leq\left\|x_{n}-p\right\| .
$$

Set $\bar{V}=I+\mu V$. Then from (56) and (64), we obtain

$$
\begin{aligned}
& \left\|y_{n}-p\right\| \\
& =\left\|\alpha_{n}\left(u+\gamma f\left(x_{n}\right)\right)+\beta_{n} x_{n}+\left(\left(1-\beta_{n}\right) I-\alpha_{n} \bar{V}\right) W_{n} z_{n}-p\right\|
\end{aligned}
$$

$$
\begin{aligned}
& =\| \alpha_{n} u+\alpha_{n}\left(\gamma f\left(x_{n}\right)-\bar{V} p\right)+\beta_{n}\left(x_{n}-p\right) \\
& +\left(\left(1-\beta_{n}\right) I-\alpha_{n} \bar{V}\right)\left(W_{n} z_{n}-p\right) \| \\
& \leq\left(1-\beta_{n}-\alpha_{n}-\alpha_{n} \mu \bar{\gamma}\right)\left\|z_{n}-p\right\|+\beta_{n}\left\|x_{n}-p\right\| \\
& +\alpha_{n}\|u\|+\alpha_{n}\left\|\gamma f\left(x_{n}\right)-\bar{V} p\right\| \\
& \leq\left(1-\beta_{n}-\alpha_{n}-\alpha_{n} \mu \bar{\gamma}\right)\left\|x_{n}-p\right\|+\beta_{n}\left\|x_{n}-p\right\| \\
& +\alpha_{n}\|u\|+\alpha_{n}\left\|\gamma f\left(x_{n}\right)-\bar{V} p\right\| \\
& \leq\left(1-\alpha_{n}-\alpha_{n} \mu \bar{\gamma}\right)\left\|x_{n}-p\right\|+\alpha_{n}\|u\| \\
& +\alpha_{n}\left(\gamma\left\|f\left(x_{n}\right)-f(p)\right\|+\|\gamma f(p)-\bar{V} p\|\right) \\
& \leq\left(1-\alpha_{n}-\alpha_{n} \mu \bar{\gamma}\right)\left\|x_{n}-p\right\|+\alpha_{n}\|u\| \\
& +\alpha_{n}\left(\gamma l\left\|x_{n}-p\right\|+\|\gamma f(p)-\bar{V} p\|\right) \\
& \leq\left[1-((1+\mu) \bar{\gamma}-\gamma l) \alpha_{n}\right]\left\|x_{n}-p\right\| \\
& +\alpha_{n}(\|\gamma f(p)-\bar{V} p\|+\|u\|) \\
& =\left[1-((1+\mu) \bar{\gamma}-\gamma l) \alpha_{n}\right]\left\|x_{n}-p\right\| \\
& +((1+\mu) \bar{\gamma}-\gamma l) \alpha_{n} \frac{\|\gamma f(p)-\bar{V} p\|+\|u\|}{(1+\mu) \bar{\gamma}-\gamma l} .
\end{aligned}
$$

Therefore, by Lemma 13 we have

$$
\begin{aligned}
\left\|x_{n+1}-p\right\| & \left\|\sigma_{n} \gamma f\left(y_{n}\right)+\left(I-\sigma_{n} \mu F\right) W_{n} y_{n}-p\right\| \\
= & \| \sigma_{n} \gamma\left(f\left(y_{n}\right)-f(p)\right)+\left(I-\sigma_{n} \mu F\right) W_{n} y_{n} \\
& \quad-\left(I-\sigma_{n} \mu F\right) W_{n} p+\sigma_{n}(\gamma f(p)-\mu F p) \| \\
\leq & \sigma_{n} \gamma\left\|f\left(y_{n}\right)-f(p)\right\| \\
& +\left\|\left(I-\sigma_{n} \mu F\right) W_{n} y_{n}-\left(I-\sigma_{n} \mu F\right) W_{n} p\right\| \\
& +\sigma_{n}\|\gamma f(p)-\mu F p\| \\
\leq & \sigma_{n} \gamma l\left\|y_{n}-p\right\|+\left(1-\sigma_{n} \tau\right)\left\|y_{n}-p\right\| \\
& +\sigma_{n}\|\gamma f(p)-\mu F p\| \\
= & \left(1-\sigma_{n}(\tau-\gamma l)\right)\left\|y_{n}-p\right\|+\sigma_{n}\|\gamma f(p)-\mu F p\| \\
\leq & \left(1-\sigma_{n}(\tau-\gamma l)\right) \\
& +\sigma_{n}\|\gamma f(p)-\mu F p\| \\
& \times\left[\begin{array}{l}
\left(1-((1+\mu) \bar{\gamma}-\gamma l) \alpha_{n}\right)\left\|x_{n}-p\right\| \\
\end{array}\right.
\end{aligned}
$$




$$
\begin{aligned}
\leq & \left(1-\sigma_{n}(\tau-\gamma l)\right) \max \left\{\left\|x_{n}-p\right\|, \frac{\|\gamma f(p)-\bar{V} p\|+\|u\|}{(1+\mu) \bar{\gamma}-\gamma l}\right\} \\
& +\sigma_{n}\|\gamma f(p)-\mu F p\| \\
= & \left(1-\sigma_{n}(\tau-\gamma l)\right) \max \left\{\left\|x_{n}-p\right\|, \frac{\|\gamma f(p)-\bar{V} p\|+\|u\|}{(1+\mu) \bar{\gamma}-\gamma l}\right\} \\
& +\sigma_{n}(\tau-\gamma l) \frac{\|\gamma f(p)-\mu F p\|}{\tau-\gamma l} \\
\leq & \max \left\{\left\|x_{n}-p\right\|, \frac{\|\gamma f(p)-\bar{V} p\|+\|u\|}{(1+\mu) \bar{\gamma}-\gamma l}, \frac{\|\gamma f(p)-\mu F p\|}{\tau-\gamma l}\right\} .
\end{aligned}
$$

By induction, we get

$$
\begin{aligned}
& \left\|x_{n}-p\right\| \\
& \leq \max \left\{\left\|x_{0}-p\right\|, \frac{\|\gamma f(p)-\bar{V} p\|+\|u\|}{(1+\mu) \bar{\gamma}-\gamma l}, \frac{\|\gamma f(p)-\mu F p\|}{\tau-\gamma l}\right\} .
\end{aligned}
$$

Therefore, $\left\{x_{n}\right\}$ is bounded and so are the sequences $\left\{u_{n}\right\},\left\{z_{n}\right\},\left\{y_{n}\right\},\left\{f\left(x_{n}\right)\right\},\left\{f\left(y_{n}\right)\right\},\left\{W_{n} x_{n}\right\}$, and $\left\{W_{n} z_{n}\right\}$.

Step 2. We show that $\left\|x_{n+1}-x_{n}\right\| \rightarrow 0$ as $n \rightarrow \infty$.

Indeed, define

$$
y_{n}=\beta_{n} x_{n}+\left(1-\beta_{n}\right) w_{n}, \quad \forall n \geq 1 .
$$

Then from the definition of $w_{n}$, we obtain

$$
\begin{aligned}
& w_{n+1}-w_{n} \\
&=\frac{y_{n+1}-\beta_{n+1} x_{n+1}}{1-\beta_{n+1}}-\frac{y_{n}-\beta_{n} x_{n}}{1-\beta_{n}} \\
&=\frac{\alpha_{n+1}\left(u+\gamma f\left(x_{n+1}\right)\right)+\left(\left(1-\beta_{n+1}\right) I-\alpha_{n+1} \bar{V}\right) W_{n+1} z_{n+1}}{1-\beta_{n+1}} \\
&=\frac{\alpha_{n}\left(u+\gamma f\left(x_{n}\right)\right)+\left(\left(1-\beta_{n}\right) I-\alpha_{n} \bar{V}\right) W_{n} z_{n}}{1-\beta_{n}} \\
&=\frac{\alpha_{n+1}}{1-\beta_{n+1}}\left(u+\gamma f\left(x_{n+1}\right)\right) \\
&+\frac{\alpha_{n}}{1-\beta_{n}}\left(u+\gamma f\left(x_{n}\right)\right)+W_{n+1} z_{n+1}-W_{n} z_{n} \\
&+\frac{\alpha_{n}}{1-\beta_{n}} \bar{V} W_{n} z_{n}-\frac{\alpha_{n+1}}{1-\beta_{n+1}} W_{n+1} z_{n+1} \\
&= \frac{\alpha_{n+1}}{1-\beta_{n+1}}\left[u+\gamma f\left(x_{n+1}\right)-\bar{V} W_{n+1} z_{n+1}\right] \\
&+\frac{\alpha_{n}}{1-\beta_{n}}\left[\bar{V} W_{n} z_{n}-u-\gamma f\left(x_{n}\right)\right] \\
& W_{n+1} z_{n+1}-W_{n+1} z_{n}+W_{n+1} z_{n}-W_{n} z_{n} .
\end{aligned}
$$

It follows that

$$
\begin{aligned}
& \left\|w_{n+1}-w_{n}\right\| \\
& \leq \frac{\alpha_{n+1}}{1-\beta_{n+1}}\left(\|u\|+\left\|\gamma f\left(x_{n+1}\right)\right\|+\left\|\bar{V} W_{n+1} z_{n+1}\right\|\right) \\
& \quad+\frac{\alpha_{n}}{1-\beta_{n}}\left(\left\|\bar{V} W_{n} z_{n}\right\|+\|u\|+\left\|\gamma f\left(x_{n}\right)\right\|\right) \\
& \quad+\left\|W_{n+1} z_{n+1}-W_{n+1} z_{n}\right\|+\left\|W_{n+1} z_{n}-W_{n} z_{n}\right\| \\
& \leq \frac{\alpha_{n+1}}{1-\beta_{n+1}}\left(\|u\|+\left\|\gamma f\left(x_{n+1}\right)\right\|+\left\|\bar{V} W_{n+1} z_{n+1}\right\|\right) \\
& \quad+\frac{\alpha_{n}}{1-\beta_{n}}\left(\left\|\bar{V} W_{n} z_{n}\right\|+\|u\|+\left\|\gamma f\left(x_{n}\right)\right\|\right) \\
& \quad+\left\|W_{n+1} z_{n}-W_{n} z_{n}\right\|+\left\|z_{n+1}-z_{n}\right\| .
\end{aligned}
$$

From (15), since $W_{n}, T_{n}$, and $U_{n, i}$ are all nonexpansive, we have

$$
\begin{aligned}
\left\|W_{n+1} z_{n}-W_{n} z_{n}\right\| & =\left\|\lambda_{1} T_{1} U_{n+1,2} z_{n}-\lambda_{1} T_{1} U_{n, 2} z_{n}\right\| \\
& \leq \lambda_{1}\left\|U_{n+1,2} z_{n}-U_{n, 2} z_{n}\right\| \\
& =\lambda_{1}\left\|\lambda_{2} T_{2} U_{n+1,3} z_{n}-\lambda_{2} T_{2} U_{n, 3} z_{n}\right\| \\
& \leq \lambda_{1} \lambda_{2}\left\|U_{n+1,3} z_{n}-U_{n, 3} z_{n}\right\| \\
& \vdots \\
& \leq \lambda_{1} \lambda_{2} \cdots \lambda_{n}\left\|U_{n+1, n+1} z_{n}-U_{n, n+1} z_{n}\right\| \\
& \leq M \prod_{i=1}^{n} \lambda_{i}, \\
\left\|W_{n+1} y_{n}-W_{n} y_{n}\right\| & =\left\|\lambda_{1} T_{1} U_{n+1,2} y_{n}-\lambda_{1} T_{1} U_{n, 2} y_{n}\right\| \\
& \leq \lambda_{1}\left\|U_{n+1,2} y_{n}-U_{n, 2} y_{n}\right\| \\
& =\lambda_{1}\left\|\lambda_{2} T_{2} U_{n+1,3} y_{n}-\lambda_{2} T_{2} U_{n, 3} y_{n}\right\| \\
& \leq \lambda_{1} \lambda_{2}\left\|U_{n+1,3} y_{n}-U_{n, 3} y_{n}\right\| \\
& \leq M \prod_{i=1}^{n} \lambda_{i}, \\
& \leq \lambda_{1} \lambda_{2} \cdots \lambda_{n}\left\|U_{n+1, n+1} y_{n}-U_{n, n+1} y_{n}\right\| \\
& \\
& \\
&
\end{aligned}
$$

where $M$ is a constant such that

$$
\begin{aligned}
& \sup _{n \geq 1}\left\{\left\|U_{n+1, n+1} z_{n}\right\|+\left\|U_{n, n+1} z_{n}\right\|\right\} \leq M, \\
& \sup _{n \geq 1}\left\{\left\|U_{n+1, n+1} y_{n}\right\|+\left\|U_{n, n+1} y_{n}\right\|\right\} \leq M .
\end{aligned}
$$

On the other hand, we estimate $\left\|z_{n+1}-z_{n}\right\|$. Taking into consideration that $0<\liminf _{n \rightarrow \infty} \beta_{n} \leq \limsup _{n \rightarrow \infty} \beta_{n}<1$ 
and $0<\liminf _{n \rightarrow \infty} r_{n} \leq \limsup _{n \rightarrow \infty} r_{n}<2 \zeta$, we may assume, without loss of generality, that $\left\{r_{n}\right\} \subset[c, d] \subset(0,2 \zeta)$ and $\left\{\beta_{n}\right\} \subset[e, f] \subset(0,1)$. Utilizing Remark 3 and Lemma 14 , we have

$$
\begin{aligned}
& \left\|z_{n+1}-z_{n}\right\|^{2} \\
& =\| J_{R_{N}, \mu_{N}}\left(I-\mu_{N} B_{N}\right) \Lambda^{N-1} u_{n+1} \\
& \quad \quad-J_{R_{N}, \mu_{N}}\left(I-\mu_{N} B_{N}\right) \Lambda^{N-1} u_{n} \|^{2} \\
& \leq\left\|\left(I-\mu_{N} B_{N}\right) \Lambda^{N-1} u_{n+1}-\left(I-\mu_{N} B_{N}\right) \Lambda^{N-1} u_{n}\right\|^{2} \\
& =\|\left(\Lambda^{N-1} u_{n+1}-\Lambda^{N-1} u_{n}\right) \\
& \quad-\mu_{N}\left(B_{N} \Lambda^{N-1} u_{n+1}-B_{N} \Lambda^{N-1} u_{n}\right) \|^{2} \\
& \leq\left\|\Lambda^{N-1} u_{n+1}-\Lambda^{N-1} u_{n}\right\|^{2}+\mu_{N}\left(\mu_{N}-2 \eta_{N}\right) \\
& \quad \times\left\|B_{N} \Lambda^{N-1} u_{n+1}-B_{N} \Lambda^{N-1} u_{n}\right\|^{2} \\
& \leq\left\|\Lambda^{N-1} u_{n+1}-\Lambda^{N-1} u_{n}\right\|^{2}
\end{aligned}
$$$$
\vdots
$$$$
\leq\left\|\Lambda^{0} u_{n+1}-\Lambda^{0} u_{n}\right\|^{2}
$$$$
=\left\|u_{n+1}-u_{n}\right\|^{2} \text {, }
$$$$
\left\|\left(I-r_{n+1} A\right) x_{n+1}-\left(I-r_{n} A\right) x_{n}\right\|
$$$$
=\left\|x_{n+1}-x_{n}-r_{n+1}\left(A x_{n+1}-A x_{n}\right)+\left(r_{n}-r_{n+1}\right) A x_{n}\right\|
$$$$
\leq\left\|x_{n+1}-x_{n}-r_{n+1}\left(A x_{n+1}-A x_{n}\right)\right\|+\left|r_{n+1}-r_{n}\right|\left\|A x_{n}\right\|
$$$$
\leq\left\|x_{n+1}-x_{n}\right\|+\left|r_{n+1}-r_{n}\right|\left\|A x_{n}\right\| \text {, }
$$$$
\left\|u_{n+1}-u_{n}\right\|
$$

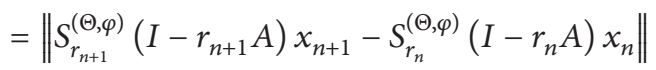

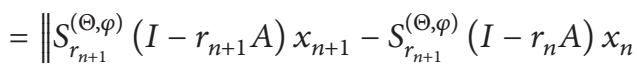$$
+S_{r_{n+1}}^{(\Theta, \varphi)}\left(I-r_{n} A\right) x_{n}-S_{r_{n}}^{(\Theta, \varphi)}\left(I-r_{n} A\right) x_{n} \|
$$$$
\leq\left\|S_{r_{n+1}}^{(\Theta, \varphi)}\left(I-r_{n+1} A\right) x_{n+1}-S_{r_{n+1}}^{(\Theta, \varphi)}\left(I-r_{n} A\right) x_{n}\right\|
$$$$
+\left\|S_{r_{n+1}}^{(\Theta, \varphi)}\left(I-r_{n} A\right) x_{n}-S_{r_{n}}^{(\Theta, \varphi)}\left(I-r_{n} A\right) x_{n}\right\|
$$$$
\leq\left\|\left(I-r_{n+1} A\right) x_{n+1}-\left(I-r_{n} A\right) x_{n}\right\|
$$$$
+\left\|S_{r_{n+1}}^{(\Theta, \varphi)}\left(I-r_{n} A\right) x_{n}-S_{r_{n}}^{(\Theta, \varphi)}\left(I-r_{n} A\right) x_{n}\right\|
$$$$
\leq\left\|x_{n+1}-x_{n}\right\|+\left|r_{n+1}-r_{n}\right|\left\|A x_{n}\right\|
$$$$
+\left\|S_{r_{n+1}}^{(\Theta, \varphi)}\left(I-r_{n} A\right) x_{n}-S_{r_{n}}^{(\Theta, \varphi)}\left(I-r_{n} A\right) x_{n}\right\|
$$

$$
\begin{aligned}
& \leq\left\|x_{n+1}-x_{n}\right\|+\left|r_{n+1}-r_{n}\right|\left\|A x_{n}\right\| \\
& +\frac{\left|r_{n+1}-r_{n}\right|}{r_{n+1}} \cdot \frac{\nu}{\sigma} \\
& \times\left\|S_{r_{n+1}}^{(\Theta, \varphi)}\left(I-r_{n} A\right) x_{n}-\left(I-r_{n} A\right) x_{n}\right\| \\
& \leq\left\|x_{n+1}-x_{n}\right\|+\left|r_{n+1}-r_{n}\right| \\
& \times\left(\left\|A x_{n}\right\|\right. \\
& \left.+\frac{v}{c \sigma}\left\|S_{r_{n+1}}^{(\Theta, \varphi)}\left(I-r_{n} A\right) x_{n}-\left(I-r_{n} A\right) x_{n}\right\|\right) \\
& \leq\left\|x_{n+1}-x_{n}\right\|+\left|r_{n+1}-r_{n}\right| M_{1} \text {, }
\end{aligned}
$$

where $\sup _{n \geq 1}\left\{\left\|A x_{n}\right\|+(\nu / c \sigma) \| S_{r_{n+1}}^{(\Theta, \varphi)}\left(I-r_{n} A\right) x_{n}-\left(I-r_{n} A\right)\right.$ $\left.x_{n} \|\right\} \leq M_{1}$ for some $M_{1}>0$.

Note that

$$
\begin{aligned}
y_{n+1}-y_{n}= & \beta_{n}\left(x_{n+1}-x_{n}\right)+\left(\beta_{n+1}-\beta_{n}\right)\left(x_{n+1}-w_{n+1}\right) \\
& +\left(1-\beta_{n}\right)\left(w_{n+1}-w_{n}\right), \\
x_{n+2}-x_{n+1} & =\sigma_{n} \gamma\left(f\left(y_{n+1}\right)-f\left(y_{n}\right)\right)+\left(\sigma_{n+1}-\sigma_{n}\right) \\
& \times\left(\gamma f\left(y_{n+1}\right)-\mu F W_{n+1} y_{n+1}\right) \\
& +\left(I-\sigma_{n} \mu F\right) W_{n+1} y_{n+1}-\left(I-\sigma_{n} \mu F\right) W_{n} y_{n} .
\end{aligned}
$$

Hence, from (70), (71), (74), and (75) it follows that

$$
\begin{aligned}
& \left\|y_{n+1}-y_{n}\right\| \\
& \leq \beta_{n}\left\|x_{n+1}-x_{n}\right\|+\left|\beta_{n+1}-\beta_{n}\right|\left\|x_{n+1}-w_{n+1}\right\| \\
& \quad+\left(1-\beta_{n}\right)\left\|w_{n+1}-w_{n}\right\| \\
& \leq \beta_{n}\left\|x_{n+1}-x_{n}\right\|+\left|\beta_{n+1}-\beta_{n}\right|\left\|x_{n+1}-w_{n+1}\right\| \\
& \quad+\left(1-\beta_{n}\right)\left[\frac { \alpha _ { n + 1 } } { 1 - \beta _ { n + 1 } } \left(\|u\|+\left\|\gamma f\left(x_{n+1}\right)\right\|\right.\right. \\
& \left.+\left\|\bar{V} W_{n+1} z_{n+1}\right\|\right) \\
& \quad+\frac{\alpha_{n}}{1-\beta_{n}}\left(\left\|\bar{V} W_{n} z_{n}\right\|+\|u\|+\left\|\gamma f\left(x_{n}\right)\right\|\right) \\
& \left.\quad+\left\|W_{n+1} z_{n}-W_{n} z_{n}\right\|+\left\|z_{n+1}-z_{n}\right\|\right] \\
& \leq \beta_{n}\left\|x_{n+1}-x_{n}\right\|+\left|\beta_{n+1}-\beta_{n}\right|\left\|x_{n+1}-w_{n+1}\right\| \\
& +\left(1-\beta_{n}\right)\left[\frac { \alpha _ { n + 1 } } { 1 - \beta _ { n + 1 } } \left(\|u\|+\left\|\gamma f\left(x_{n+1}\right)\right\|\right.\right.
\end{aligned}
$$




$$
\begin{aligned}
& \left.+\left\|\bar{V} W_{n+1} z_{n+1}\right\|\right) \\
& +\frac{\alpha_{n}}{1-\beta_{n}}\left(\left\|\bar{V} W_{n} z_{n}\right\|+\|u\|+\left\|\gamma f\left(x_{n}\right)\right\|\right) \\
& \left.+M \prod_{i=1}^{n} \lambda_{i}+\left\|z_{n+1}-z_{n}\right\|\right] \\
& \leq \beta_{n}\left\|x_{n+1}-x_{n}\right\|+\left|\beta_{n+1}-\beta_{n}\right|\left\|x_{n+1}-w_{n+1}\right\| \\
& +\left(1-\beta_{n}\right)\left[\frac { \alpha _ { n + 1 } } { 1 - \beta _ { n + 1 } } \left(\|u\|+\left\|\gamma f\left(x_{n+1}\right)\right\|\right.\right. \\
& \left.+\left\|\bar{V} W_{n+1} z_{n+1}\right\|\right) \\
& +\frac{\alpha_{n}}{1-\beta_{n}}\left(\left\|\bar{V} W_{n} z_{n}\right\|+\|u\|+\left\|\gamma f\left(x_{n}\right)\right\|\right) \\
& \left.+M \prod_{i=1}^{n} \lambda_{i}+\left\|u_{n+1}-u_{n}\right\|\right] \\
& \leq \beta_{n}\left\|x_{n+1}-x_{n}\right\|+\left|\beta_{n+1}-\beta_{n}\right|\left\|x_{n+1}-w_{n+1}\right\| \\
& +\left(1-\beta_{n}\right)\left[\frac { \alpha _ { n + 1 } } { 1 - \beta _ { n + 1 } } \left(\|u\|+\left\|\gamma f\left(x_{n+1}\right)\right\|\right.\right. \\
& \left.+\left\|\bar{V} W_{n+1} z_{n+1}\right\|\right) \\
& +\frac{\alpha_{n}}{1-\beta_{n}}\left(\left\|\bar{V} W_{n} z_{n}\right\|+\|u\|+\left\|\gamma f\left(x_{n}\right)\right\|\right) \\
& +M \prod_{i=1}^{n} \lambda_{i}+\left\|x_{n+1}-x_{n}\right\| \\
& \left.+\left|r_{n+1}-r_{n}\right| M_{1}\right] \\
& \leq\left\|x_{n+1}-x_{n}\right\|+\left|\beta_{n+1}-\beta_{n}\right| M_{2} \\
& +M_{2}\left(\alpha_{n+1}+\alpha_{n}\right)+M_{2} \prod_{i=1}^{n} \lambda_{i}+\left|r_{n+1}-r_{n}\right| M_{2},
\end{aligned}
$$

where $\sup _{n \geq 1}\left\{(1 /(1-f))\left(\left\|\bar{V} W_{n} z_{n}\right\|+\|u\|+\left\|\gamma f\left(x_{n}\right)\right\|\right)+\| x_{n}-\right.$ $\left.w_{n} \|+M_{1}+M\right\} \leq M_{2}$ for some $M_{2}>0$. So, utilizing Lemma 13 we obtain from (72) and (77) that

$$
\begin{aligned}
& \left\|x_{n+2}-x_{n+1}\right\| \\
& \leq \sigma_{n} \gamma\left\|f\left(y_{n+1}\right)-f\left(y_{n}\right)\right\|+\left|\sigma_{n+1}-\sigma_{n}\right| \\
& \quad \times\left\|\gamma f\left(y_{n+1}\right)-\mu F W_{n+1} y_{n+1}\right\| \\
& \quad+\left\|\left(I-\sigma_{n} \mu F\right) W_{n+1} y_{n+1}-\left(I-\sigma_{n} \mu F\right) W_{n} y_{n}\right\| \\
& \leq \sigma_{n} \gamma l\left\|y_{n+1}-y_{n}\right\|+\left|\sigma_{n+1}-\sigma_{n}\right| \\
& \quad \times\left\|\gamma f\left(y_{n+1}\right)-\mu F W_{n+1} y_{n+1}\right\| \\
& \quad+\left(1-\sigma_{n} \tau\right)\left\|W_{n+1} y_{n+1}-W_{n} y_{n}\right\|
\end{aligned}
$$

$$
\begin{aligned}
& \leq \sigma_{n} \gamma l\left\|y_{n+1}-y_{n}\right\|+\left|\sigma_{n+1}-\sigma_{n}\right| \\
& \times\left\|\gamma f\left(y_{n+1}\right)-\mu F W_{n+1} y_{n+1}\right\| \\
& +\left(1-\sigma_{n} \tau\right)\left(\left\|W_{n+1} y_{n+1}-W_{n+1} y_{n}\right\|\right. \\
& \left.+\left\|W_{n+1} y_{n}-W_{n} y_{n}\right\|\right) \\
& \leq \sigma_{n} \gamma l\left\|y_{n+1}-y_{n}\right\|+\left|\sigma_{n+1}-\sigma_{n}\right| \\
& \times\left\|\gamma f\left(y_{n+1}\right)-\mu F W_{n+1} y_{n+1}\right\| \\
& +\left(1-\sigma_{n} \tau\right)\left(\left\|y_{n+1}-y_{n}\right\|+M \prod_{i=1}^{n} \lambda_{i}\right) \\
& \leq\left(1-\sigma_{n}(\tau-\gamma l)\right)\left\|y_{n+1}-y_{n}\right\|+\left|\sigma_{n+1}-\sigma_{n}\right| \\
& \times\left\|\gamma f\left(y_{n+1}\right)-\mu F W_{n+1} y_{n+1}\right\|+M \prod_{i=1}^{n} \lambda_{i} \\
& \leq\left(1-\sigma_{n}(\tau-\gamma l)\right) \\
& \times\left[\left\|x_{n+1}-x_{n}\right\|\right. \\
& +\left|\beta_{n+1}-\beta_{n}\right| M_{2}+M_{2}\left(\alpha_{n+1}+\alpha_{n}\right) \\
& +M_{2} \prod_{i=1}^{n} \lambda_{i} \\
& \left.+\left|r_{n+1}-r_{n}\right| M_{2}\right]+\left|\sigma_{n+1}-\sigma_{n}\right| \\
& \times\left\|\gamma f\left(y_{n+1}\right)-\mu F W_{n+1} y_{n+1}\right\|+M \prod_{i=1}^{n} \lambda_{i} \\
& \leq\left(1-\sigma_{n}(\tau-\gamma l)\right)\left\|x_{n+1}-x_{n}\right\| \\
& +\left|\beta_{n+1}-\beta_{n}\right| M_{3}+M_{3}\left(\alpha_{n+1}+\alpha_{n}\right)+M_{3} \prod_{i=1}^{n} \lambda_{i} \\
& +\left|r_{n+1}-r_{n}\right| M_{3}+\left|\sigma_{n+1}-\sigma_{n}\right| M_{3} \\
& +M_{3} \prod_{i=1}^{n} \lambda_{i} \\
& =\left(1-\sigma_{n}(\tau-\gamma l)\right)\left\|x_{n+1}-x_{n}\right\| \\
& +\left(\left|\beta_{n+1}-\beta_{n}\right|+\left|r_{n+1}-r_{n}\right|+\left|\sigma_{n+1}-\sigma_{n}\right|\right) M_{3} \\
& +2 M_{3} \prod_{i=1}^{n} \lambda_{i}+M_{3}\left(\alpha_{n+1}+\alpha_{n}\right) \\
& \leq\left(1-\sigma_{n}(\tau-\gamma l)\right)\left\|x_{n+1}-x_{n}\right\| \\
& +\left(\left|\beta_{n+1}-\beta_{n}\right|+\left|r_{n+1}-r_{n}\right|+\left|\sigma_{n+1}-\sigma_{n}\right|\right) M_{3} \\
& +2 M_{3} b^{n} \\
& +\sigma_{n}(\tau-\gamma l) \frac{M_{3}}{\tau-\gamma l} \cdot \frac{\alpha_{n}}{\sigma_{n}}\left(\frac{\alpha_{n+1}}{\alpha_{n}}+1\right),
\end{aligned}
$$


where $\sup _{n \geq 1}\left\{\left\|\gamma f\left(y_{n}\right)-\mu F W_{n} y_{n}\right\|+M_{2}+M\right\} \leq M_{3}$ for some $M_{3}>0$. Applying Lemma 19 to (78), we deduce from conditions (iii) and (v) that

$$
\lim _{n \rightarrow \infty}\left\|x_{n+1}-x_{n}\right\|=0
$$

Step 3. $\left\|x_{n}-y_{n}\right\| \rightarrow 0$ as $n \rightarrow \infty$. Indeed, utilizing Lemmas 4 and 5(b) we obtain from (56) and (64) that

$$
\begin{aligned}
&\left\|y_{n}-p\right\|^{2} \\
&=\| \alpha_{n}\left(\left(u+\gamma f\left(x_{n}\right)\right)-(I+\mu V) W_{n} z_{n}\right) \\
& \quad+\beta_{n}\left(x_{n}-p\right)+\left(1-\beta_{n}\right)\left(W_{n} z_{n}-p\right) \|^{2} \\
& \leq\left\|\beta_{n}\left(x_{n}-p\right)+\left(1-\beta_{n}\right)\left(W_{n} z_{n}-p\right)\right\|^{2} \\
&+2 \alpha_{n}\left\langle\left(u+\gamma f\left(x_{n}\right)\right)-(I+\mu V) W_{n} z_{n}, y_{n}-p\right\rangle \\
&= \beta_{n}\left\|x_{n}-p\right\|^{2}+\left(1-\beta_{n}\right)\left\|W_{n} z_{n}-p\right\|^{2} \\
&-\beta_{n}\left(1-\beta_{n}\right)\left\|x_{n}-W_{n} z_{n}\right\|^{2} \\
&+2 \alpha_{n}\left\|\left(u+\gamma f\left(x_{n}\right)\right)-(I+\mu V) W_{n} z_{n}\right\|\left\|y_{n}-p\right\| \\
& \leq \beta_{n}\left\|x_{n}-p\right\|^{2}+\left(1-\beta_{n}\right)\left\|z_{n}-p\right\|^{2} \\
&-\beta_{n}\left(1-\beta_{n}\right)\left\|x_{n}-W_{n} z_{n}\right\|^{2} \\
&+2 \alpha_{n}\left\|\left(u+\gamma f\left(x_{n}\right)\right)-(I+\mu V) W_{n} z_{n}\right\|\left\|y_{n}-p\right\| \\
& \leq \beta_{n}\left\|x_{n}-p\right\|^{2}+\left(1-\beta_{n}\right)\left\|x_{n}-p\right\|^{2} \\
&-\beta_{n}\left(1-\beta_{n}\right)\left\|x_{n}-W_{n} z_{n}\right\|^{2} \\
&+2 \alpha_{n}\left\|\left(u+\gamma f\left(x_{n}\right)\right)-(I+\mu V) W_{n} z_{n}\right\|\left\|y_{n}-p\right\| \\
&=\left\|x_{n}-p\right\|^{2}-\beta_{n}\left(1-\beta_{n}\right)\left\|x_{n}-W_{n} z_{n}\right\|^{2} \\
&+2 \alpha_{n}\left\|\left(u+\gamma f\left(x_{n}\right)\right)-(I+\mu V) W_{n} z_{n}\right\|\left\|y_{n}-p\right\| .
\end{aligned}
$$

Utilizing Lemmas 4 and 13, we conclude from (56) and (80) that

$$
\begin{aligned}
& \left\|x_{n+1}-p\right\|^{2} \\
& =\| \sigma_{n} \gamma\left(f\left(y_{n}\right)-f(p)\right)+\left(I-\sigma_{n} \mu F\right) W_{n} y_{n} \\
& -\left(I-\sigma_{n} \mu F\right) W_{n} p+\sigma_{n}(\gamma f(p)-\mu F p) \|^{2} \\
& \leq \| \sigma_{n} \gamma\left(f\left(y_{n}\right)-f(p)\right)+\left(I-\sigma_{n} \mu F\right) W_{n} y_{n} \\
& -\left(I-\sigma_{n} \mu F\right) W_{n} p \|^{2} \\
& +2 \sigma_{n}\left\langle\gamma f(p)-\mu F p, x_{n+1}-p\right\rangle \\
& \leq\left[\sigma_{n} \gamma l\left\|y_{n}-p\right\|+\left(1-\sigma_{n} \tau\right)\left\|y_{n}-p\right\|\right]^{2} \\
& +2 \sigma_{n}\left\langle\gamma f(p)-\mu F p, x_{n+1}-p\right\rangle
\end{aligned}
$$

$$
\begin{aligned}
= & \left(1-\sigma_{n}(\tau-\gamma l)\right)^{2}\left\|y_{n}-p\right\|^{2} \\
& +2 \sigma_{n}\|\gamma f(p)-\mu F p\|\left\|x_{n+1}-p\right\| \\
\leq & \left\|y_{n}-p\right\|^{2}+2 \sigma_{n}\|\gamma f(p)-\mu F p\|\left\|x_{n+1}-p\right\| \\
\leq & \left\|x_{n}-p\right\|^{2}-\beta_{n}\left(1-\beta_{n}\right)\left\|x_{n}-W_{n} z_{n}\right\|^{2} \\
& +2 \alpha_{n}\left\|\left(u+\gamma f\left(x_{n}\right)\right)-(I+\mu V) W_{n} z_{n}\right\|\left\|y_{n}-p\right\| \\
& +2 \sigma_{n}\|\gamma f(p)-\mu F p\|\left\|x_{n+1}-p\right\|,
\end{aligned}
$$

which leads to

$$
\begin{aligned}
e & (1-f)\left\|x_{n}-W_{n} z_{n}\right\|^{2} \\
\leq & \beta_{n}\left(1-\beta_{n}\right)\left\|x_{n}-W_{n} z_{n}\right\|^{2} \\
\leq & \left\|x_{n}-p\right\|^{2}-\left\|x_{n+1}-p\right\|^{2} \\
& +2 \alpha_{n}\left\|\left(u+\gamma f\left(x_{n}\right)\right)-(I+\mu V) W_{n} z_{n}\right\|\left\|y_{n}-p\right\| \\
& +2 \sigma_{n}\|\gamma f(p)-\mu F p\|\left\|x_{n+1}-p\right\| \\
\leq & \left\|x_{n}-x_{n+1}\right\|\left(\left\|x_{n}-p\right\|+\left\|x_{n+1}-p\right\|\right) \\
& +2 \alpha_{n}\left\|\left(u+\gamma f\left(x_{n}\right)\right)-(I+\mu V) W_{n} z_{n}\right\|\left\|y_{n}-p\right\| \\
& +2 \sigma_{n}\|\gamma f(p)-\mu F p\|\left\|x_{n+1}-p\right\| .
\end{aligned}
$$

Since $\lim _{n \rightarrow \infty}\left\|x_{n}-x_{n+1}\right\|=0, \lim _{n \rightarrow \infty} \alpha_{n}=0$, and $\lim _{n \rightarrow \infty} \sigma_{n}=0$, we deduce from the boundedness of $\left\{x_{n}\right\},\left\{y_{n}\right\},\left\{f\left(x_{n}\right)\right\}$ and $\left\{W_{n} z_{n}\right\}$ that

$$
\lim _{n \rightarrow \infty}\left\|x_{n}-W_{n} z_{n}\right\|=0
$$

Note that

$$
\begin{aligned}
& \left\|y_{n}-x_{n}\right\| \\
& =\| \alpha_{n}\left(\left(u+\gamma f\left(x_{n}\right)\right)-(I+\mu V) W_{n} z_{n}\right) \\
& \quad+\left(1-\beta_{n}\right)\left(W_{n} z_{n}-x_{n}\right) \| \\
& \leq \alpha_{n}\left\|\left(u+\gamma f\left(x_{n}\right)\right)-(I+\mu V) W_{n} z_{n}\right\| \\
& \quad+\left(1-\beta_{n}\right)\left\|W_{n} z_{n}-x_{n}\right\| \\
& \leq \alpha_{n}\left\|\left(u+\gamma f\left(x_{n}\right)\right)-(I+\mu V) W_{n} z_{n}\right\| \\
& \quad+\left\|W_{n} z_{n}-x_{n}\right\| .
\end{aligned}
$$

So, it follows from (83) and $\lim _{n \rightarrow \infty} \alpha_{n}=0$ that

$$
\lim _{n \rightarrow \infty}\left\|x_{n}-y_{n}\right\|=0 \text {. }
$$


Step 4. $\left\|x_{n}-u_{n}\right\| \rightarrow 0,\left\|u_{n}-z_{n}\right\| \rightarrow 0$ and $\left\|z_{n}-W z_{n}\right\| \rightarrow 0$ as $n \rightarrow \infty$. Indeed, for $p \in \Omega$, we find that

$$
\begin{aligned}
\| u_{n} & -p \|^{2} \\
& =\left\|S_{r_{n}}^{(\Theta, \varphi)}\left(I-r_{n} A\right) x_{n}-S_{r_{n}}^{(\Theta, \varphi)}\left(I-r_{n} A\right) p\right\|^{2} \\
& \leq\left\|\left(I-r_{n} A\right) x_{n}-\left(I-r_{n} A\right) p\right\|^{2} \\
& =\left\|x_{n}-p-r_{n}\left(A x_{n}-A p\right)\right\|^{2} \\
& \leq\left\|x_{n}-p\right\|^{2}+r_{n}\left(r_{n}-2 \zeta\right)\left\|A x_{n}-A p\right\|^{2} .
\end{aligned}
$$

From (56), (63), and (86), we obtain

$$
\begin{aligned}
\left\|y_{n}-p\right\|^{2} & \\
= & \| \alpha_{n}\left(\left(u+\gamma f\left(x_{n}\right)\right)-(I+\mu V) W_{n} z_{n}\right) \\
& +\beta_{n}\left(x_{n}-p\right)+\left(1-\beta_{n}\right)\left(W_{n} z_{n}-p\right) \|^{2} \\
\leq & \left\|\beta_{n}\left(x_{n}-p\right)+\left(1-\beta_{n}\right)\left(W_{n} z_{n}-p\right)\right\|^{2} \\
& +2 \alpha_{n}\left\langle\left(u+\gamma f\left(x_{n}\right)\right)-(I+\mu V) W_{n} z_{n}, y_{n}-p\right\rangle \\
\leq & \beta_{n}\left\|x_{n}-p\right\|^{2}+\left(1-\beta_{n}\right)\left\|W_{n} z_{n}-p\right\|^{2} \\
& +2 \alpha_{n}\left\|\left(u+\gamma f\left(x_{n}\right)\right)-(I+\mu V) W_{n} z_{n}\right\|\left\|y_{n}-p\right\| \\
\leq & \beta_{n}\left\|x_{n}-p\right\|^{2}+\left(1-\beta_{n}\right)\left\|z_{n}-p\right\|^{2} \\
& +2 \alpha_{n}\left\|\left(u+\gamma f\left(x_{n}\right)\right)-(I+\mu V) W_{n} z_{n}\right\|\left\|y_{n}-p\right\| \\
\leq & \beta_{n}\left\|x_{n}-p\right\|^{2}+\left(1-\beta_{n}\right)\left\|u_{n}-p\right\|^{2} \\
& +2 \alpha_{n}\left\|\left(u+\gamma f\left(x_{n}\right)\right)-(I+\mu V) W_{n} z_{n}\right\|\left\|y_{n}-p\right\| \\
\leq & \beta_{n}\left\|x_{n}-p\right\|^{2}+\left(1-\beta_{n}\right) \\
& \times \\
& {\left[\left\|x_{n}-p\right\|^{2}+r_{n}\left(r_{n}-2 \zeta\right)\left\|A x_{n}-A p\right\|^{2}\right] } \\
& +2 \alpha_{n}\left\|\left(u+\gamma f\left(x_{n}\right)\right)-(I+\mu V) W_{n} z_{n}\right\| \\
& \times\left\|y_{n}-p\right\| \\
= & \left\|x_{n}-p\right\|^{2}+\left(1-\beta_{n}\right) r_{n}\left(r_{n}-2 \zeta\right)\left\|A x_{n}-A p\right\|^{2} \\
& +2 \alpha_{n}\left\|\left(u+\gamma f\left(x_{n}\right)\right)-(I+\mu V) W_{n} z_{n}\right\|\left\|y_{n}-p\right\|,
\end{aligned}
$$

which immediately implies that

$$
\begin{aligned}
& (1-f) c(2 \zeta-d)\left\|A x_{n}-A p\right\|^{2} \\
& \leq\left(1-\beta_{n}\right) r_{n}\left(2 \zeta-r_{n}\right)\left\|A x_{n}-A p\right\|^{2} \\
& \leq\left\|x_{n}-p\right\|^{2}-\left\|y_{n}-p\right\|^{2} \\
& \quad+2 \alpha_{n}\left\|\left(u+\gamma f\left(x_{n}\right)\right)-(I+\mu V) W_{n} z_{n}\right\|\left\|y_{n}-p\right\| \\
& \leq\left\|x_{n}-y_{n}\right\|\left(\left\|x_{n}-p\right\|+\left\|y_{n}-p\right\|\right) \\
& \quad+2 \alpha_{n}\left\|\left(u+\gamma f\left(x_{n}\right)\right)-(I+\mu V) W_{n} z_{n}\right\|\left\|y_{n}-p\right\| .
\end{aligned}
$$

From $\lim _{n \rightarrow \infty} \alpha_{n}=0$ and $\lim _{n \rightarrow \infty}\left\|x_{n}-y_{n}\right\|=0$, we have

$$
\lim _{n \rightarrow \infty}\left\|A x_{n}-A p\right\|=0
$$

Furthermore, from the firm nonexpansivity of $S_{r_{n}}^{(\Theta, \varphi)}$, we have

$$
\begin{aligned}
& \left\|u_{n}-p\right\|^{2} \\
& =\left\|S_{r_{n}}^{(\Theta, \varphi)}\left(I-r_{n} A\right) x_{n}-S_{r_{n}}^{(\Theta, \varphi)}\left(I-r_{n} A\right) p\right\|^{2} \\
& \leq\left\langle\left(I-r_{n} A\right) x_{n}-\left(I-r_{n} A\right) p, u_{n}-p\right\rangle \\
& =\frac{1}{2}\left[\left\|\left(I-r_{n} A\right) x_{n}-\left(I-r_{n} A\right) p\right\|^{2}+\left\|u_{n}-p\right\|^{2}\right. \\
& \left.\quad-\left\|\left(I-r_{n} A\right) x_{n}-\left(I-r_{n} A\right) p-\left(u_{n}-p\right)\right\|^{2}\right] \\
& \leq \frac{1}{2}\left[\left\|x_{n}-p\right\|^{2}+\left\|u_{n}-p\right\|^{2}\right. \\
& \left.\quad-\left\|x_{n}-u_{n}-r_{n}\left(A x_{n}-A p\right)\right\|^{2}\right] \\
& =\frac{1}{2}\left[\left\|x_{n}-p\right\|^{2}+\left\|u_{n}-p\right\|^{2}-\left\|x_{n}-u_{n}\right\|^{2}\right. \\
& \left.\quad+2 r_{n}\left\langle A x_{n}-A p, x_{n}-u_{n}\right\rangle-r_{n}^{2}\left\|A x_{n}-A p\right\|^{2}\right],
\end{aligned}
$$

which implies that

$$
\begin{aligned}
\left\|u_{n}-p\right\|^{2} \leq & \left\|x_{n}-p\right\|^{2}-\left\|x_{n}-u_{n}\right\|^{2} \\
& +2 r_{n}\left\|A x_{n}-A p\right\|\left\|x_{n}-u_{n}\right\| .
\end{aligned}
$$

From (87) and (91), we have

$$
\begin{aligned}
& \left\|y_{n}-p\right\|^{2} \\
& \leq \beta_{n}\left\|x_{n}-p\right\|^{2}+\left(1-\beta_{n}\right)\left\|u_{n}-p\right\|^{2} \\
& \quad+2 \alpha_{n}\left\|\left(u+\gamma f\left(x_{n}\right)\right)-(I+\mu V) W_{n} z_{n}\right\|\left\|y_{n}-p\right\| \\
& \leq \beta_{n}\left\|x_{n}-p\right\|^{2} \\
& \quad+\left(1-\beta_{n}\right)\left[\left\|x_{n}-p\right\|^{2}\right. \\
& \left.\quad-\left\|x_{n}-u_{n}\right\|^{2}+2 r_{n}\left\|A x_{n}-A p\right\|\left\|x_{n}-u_{n}\right\|\right] \\
& \quad+2 \alpha_{n}\left\|\left(u+\gamma f\left(x_{n}\right)\right)-(I+\mu V) W_{n} z_{n}\right\|\left\|y_{n}-p\right\| \\
& \leq\left\|x_{n}-p\right\|^{2}-\left(1-\beta_{n}\right)\left\|x_{n}-u_{n}\right\|^{2} \\
& \quad+2 r_{n}\left\|A x_{n}-A p\right\|\left\|x_{n}-u_{n}\right\| \\
& \quad+2 \alpha_{n}\left\|\left(u+\gamma f\left(x_{n}\right)\right)-(I+\mu V) W_{n} z_{n}\right\|\left\|y_{n}-p\right\| .
\end{aligned}
$$


It follows that

$$
\begin{aligned}
& (1-f)\left\|x_{n}-u_{n}\right\|^{2} \\
& \leq\left(1-\beta_{n}\right)\left\|x_{n}-u_{n}\right\|^{2} \\
& \leq\left\|x_{n}-p\right\|^{2}-\left\|y_{n}-p\right\|^{2} \\
& \quad+2 r_{n}\left\|A x_{n}-A p\right\|\left\|x_{n}-u_{n}\right\| \\
& \quad+2 \alpha_{n}\left\|\left(u+\gamma f\left(x_{n}\right)\right)-(I+\mu V) W_{n} z_{n}\right\|\left\|y_{n}-p\right\| \\
& \leq\left\|x_{n}-y_{n}\right\|\left(\left\|x_{n}-p\right\|+\left\|y_{n}-p\right\|\right) \\
& \quad+2 d\left\|A x_{n}-A p\right\|\left\|x_{n}-u_{n}\right\| \\
& \quad+2 \alpha_{n}\left\|\left(u+\gamma f\left(x_{n}\right)\right)-(I+\mu V) W_{n} z_{n}\right\|\left\|y_{n}-p\right\| .
\end{aligned}
$$

So, from (85), (89), and $\lim _{n \rightarrow \infty} \alpha_{n}=0$, we obtain

$$
\lim _{n \rightarrow \infty}\left\|x_{n}-u_{n}\right\|=0 \text {. }
$$

Next we show that $\lim _{n \rightarrow \infty}\left\|A_{i} \Lambda^{i} u_{n}-A_{i} p\right\|=0, i=1,2, \ldots$, $N$. Observe that

$$
\begin{aligned}
& \left\|\Lambda^{i} u_{n}-p\right\|^{2} \\
& =\left\|J_{R_{i}, \mu_{i}}\left(I-\mu_{i} B_{i}\right) \Lambda^{i-1} u_{n}-J_{R_{i}, \mu_{i}}\left(I-\mu_{i} B_{i}\right) p\right\|^{2} \\
& \leq\left\|\left(I-\mu_{i} B_{i}\right) \Lambda^{i-1} u_{n}-\left(I-\mu_{i} B_{i}\right) p\right\|^{2} \\
& \leq\left\|\Lambda^{i-1} u_{n}-p\right\|^{2}+\mu_{i}\left(\mu_{i}-2 \eta_{i}\right) \\
& \quad \times\left\|B_{i} \Lambda^{i-1} u_{n}-B_{i} p\right\|^{2} \\
& \leq\left\|u_{n}-p\right\|^{2}+\mu_{i}\left(\mu_{i}-2 \eta_{i}\right)\left\|B_{i} \Lambda^{i-1} u_{n}-B_{i} p\right\|^{2} \\
& \leq\left\|x_{n}-p\right\|^{2}+\mu_{i}\left(\mu_{i}-2 \eta_{i}\right)\left\|B_{i} \Lambda^{i-1} u_{n}-B_{i} p\right\|^{2} .
\end{aligned}
$$

Combining (87) and (95), we have

$$
\begin{aligned}
\left\|y_{n}-p\right\|^{2} & \\
\leq & \beta_{n}\left\|x_{n}-p\right\|^{2}+\left(1-\beta_{n}\right)\left\|z_{n}-p\right\|^{2} \\
& +2 \alpha_{n}\left\|\left(u+\gamma f\left(x_{n}\right)\right)-(I+\mu V) W_{n} z_{n}\right\|\left\|y_{n}-p\right\| \\
\leq & \beta_{n}\left\|x_{n}-p\right\|^{2}+\left(1-\beta_{n}\right)\left\|\Lambda^{i} u_{n}-p\right\|^{2} \\
& +2 \alpha_{n}\left\|\left(u+\gamma f\left(x_{n}\right)\right)-(I+\mu V) W_{n} z_{n}\right\|\left\|y_{n}-p\right\| \\
\leq & \beta_{n}\left\|x_{n}-p\right\|^{2}+\left(1-\beta_{n}\right) \\
& \times\left[\left\|x_{n}-p\right\|^{2}+\mu_{i}\left(\mu_{i}-2 \eta_{i}\right)\left\|B_{i} \Lambda^{i-1} u_{n}-B_{i} p\right\|^{2}\right] \\
& +2 \alpha_{n}\left\|\left(u+\gamma f\left(x_{n}\right)\right)-(I+\mu V) W_{n} z_{n}\right\|\left\|y_{n}-p\right\| \\
= & \left\|x_{n}-p\right\|^{2}+\left(1-\beta_{n}\right) \mu_{i}\left(\mu_{i}-2 \eta_{i}\right) \\
& \times\left\|B_{i} \Lambda^{i-1} u_{n}-B_{i} p\right\|^{2} \\
& +2 \alpha_{n}\left\|\left(u+\gamma f\left(x_{n}\right)\right)-(I+\mu V) W_{n} z_{n}\right\|\left\|y_{n}-p\right\|
\end{aligned}
$$

which leads to

$$
\begin{aligned}
& (1-f) \mu_{i}\left(2 \eta_{i}-\mu_{i}\right)\left\|B_{i} \Lambda^{i-1} u_{n}-B_{i} p\right\|^{2} \\
& \leq\left(1-\beta_{n}\right) \mu_{i}\left(2 \eta_{i}-\mu_{i}\right)\left\|B_{i} \Lambda^{i-1} u_{n}-B_{i} p\right\|^{2} \\
& \leq\left\|x_{n}-p\right\|^{2}-\left\|y_{n}-p\right\|^{2} \\
& \quad+2 \alpha_{n}\left\|\left(u+\gamma f\left(x_{n}\right)\right)-(I+\mu V) W_{n} z_{n}\right\|\left\|y_{n}-p\right\| \\
& \leq\left\|x_{n}-y_{n}\right\|\left(\left\|x_{n}-p\right\|+\left\|y_{n}-p\right\|\right) \\
& \quad+2 \alpha_{n}\left\|\left(u+\gamma f\left(x_{n}\right)\right)-(I+\mu V) W_{n} z_{n}\right\|\left\|y_{n}-p\right\| .
\end{aligned}
$$

Since $\mu_{i} \in\left(0,2 \eta_{i}\right), i=1,2, \ldots, N$ and $\lim _{n \rightarrow \infty} \alpha_{n}=0$, we obtain from (85) that

$$
\lim _{n \rightarrow \infty}\left\|B_{i} \Lambda^{i-1} u_{n}-B_{i} p\right\|=0, \quad \forall i \in\{1,2, \ldots, N\}
$$

By Lemmas 5(a) and (14), we obtain

$$
\begin{aligned}
& \left\|\Lambda^{i} u_{n}-p\right\|^{2} \\
& =\left\|J_{R_{i}, \mu_{i}}\left(I-\mu_{i} B_{i}\right) \Lambda^{i-1} u_{n}-J_{R_{i}, \mu_{i}}\left(I-\mu_{i} B_{i}\right) p\right\|^{2} \\
& \leq\left\langle\left(I-\mu_{i} B_{i}\right) \Lambda^{i-1} u_{n}-\left(I-\mu_{i} B_{i}\right) p, \Lambda^{i} u_{n}-p\right\rangle \\
& =\frac{1}{2}\left(\left\|\left(I-\mu_{i} B_{i}\right) \Lambda^{i-1} u_{n}-\left(I-\mu_{i} B_{i}\right) p\right\|^{2}\right. \\
& +\left\|\Lambda^{i} u_{n}-p\right\|^{2}-\|\left(I-\mu_{i} B_{i}\right) \Lambda^{i-1} u_{n} \\
& \left.-\left(I-\mu_{i} B_{i}\right) p-\left(\Lambda^{i} u_{n}-p\right) \|^{2}\right) \\
& \leq \frac{1}{2}\left(\left\|\Lambda^{i-1} u_{n}-p\right\|^{2}+\left\|\Lambda^{i} u_{n}-p\right\|^{2}\right. \\
& \left.-\left\|\Lambda^{i-1} u_{n}-\Lambda^{i} u_{n}-\mu_{i}\left(B_{i} \Lambda^{i-1} u_{n}-B_{i} p\right)\right\|^{2}\right) \\
& \leq \frac{1}{2}\left(\left\|u_{n}-p\right\|^{2}+\left\|\Lambda^{i} u_{n}-p\right\|^{2}\right. \\
& \left.-\left\|\Lambda^{i-1} u_{n}-\Lambda^{i} u_{n}-\mu_{i}\left(B_{i} \Lambda^{i-1} u_{n}-B_{i} p\right)\right\|^{2}\right) \\
& \leq \frac{1}{2}\left(\left\|x_{n}-p\right\|^{2}+\left\|\Lambda^{i} u_{n}-p\right\|^{2}\right. \\
& \left.-\left\|\Lambda^{i-1} u_{n}-\Lambda^{i} u_{n}-\mu_{i}\left(B_{i} \Lambda^{i-1} u_{n}-B_{i} p\right)\right\|^{2}\right),
\end{aligned}
$$


which implies

$$
\begin{aligned}
& \left\|\Lambda^{i} u_{n}-p\right\|^{2} \\
& \leq\left\|x_{n}-p\right\|^{2} \\
& \quad-\left\|\Lambda^{i-1} u_{n}-\Lambda^{i} u_{n}-\mu_{i}\left(B_{i} \Lambda^{i-1} u_{n}-B_{i} p\right)\right\|^{2} \\
& =\left\|x_{n}-p\right\|^{2}-\left\|\Lambda^{i-1} u_{n}-\Lambda^{i} u_{n}\right\|^{2} \\
& \quad-\mu_{i}^{2}\left\|B_{i} \Lambda^{i-1} u_{n}-B_{i} p\right\|^{2} \\
& \quad+2 \mu_{i}\left\langle\Lambda^{i-1} u_{n}-\Lambda^{i} u_{n}, B_{i} \Lambda^{i-1} u_{n}-B_{i} p\right\rangle \\
& \leq\left\|x_{n}-p\right\|^{2}-\left\|\Lambda^{i-1} u_{n}-\Lambda^{i} u_{n}\right\|^{2} \\
& \quad+2 \mu_{i}\left\|\Lambda^{i-1} u_{n}-\Lambda^{i} u_{n}\right\|\left\|B_{i} \Lambda^{i-1} u_{n}-B_{i} p\right\| .
\end{aligned}
$$

Combining (87) and (100), we have

$$
\begin{aligned}
\left\|y_{n}-p\right\|^{2} & \\
\leq & \beta_{n}\left\|x_{n}-p\right\|^{2}+\left(1-\beta_{n}\right)\left\|z_{n}-p\right\|^{2} \\
& +2 \alpha_{n}\left\|\left(u+\gamma f\left(x_{n}\right)\right)-(I+\mu V) W_{n} z_{n}\right\|\left\|y_{n}-p\right\| \\
\leq & \beta_{n}\left\|x_{n}-p\right\|^{2}+\left(1-\beta_{n}\right)\left\|\Lambda^{i} u_{n}-p\right\|^{2} \\
& +2 \alpha_{n}\left\|\left(u+\gamma f\left(x_{n}\right)\right)-(I+\mu V) W_{n} z_{n}\right\|\left\|y_{n}-p\right\| \\
\leq & \beta_{n}\left\|x_{n}-p\right\|^{2}+\left(1-\beta_{n}\right) \\
& \times\left[\left\|x_{n}-p\right\|^{2}-\left\|\Lambda^{i-1} u_{n}-\Lambda^{i} u_{n}\right\|^{2}\right. \\
& \left.+2 \mu_{i}\left\|\Lambda^{i-1} u_{n}-\Lambda^{i} u_{n}\right\|\left\|B_{i} \Lambda^{i-1} u_{n}-B_{i} p\right\|\right] \\
& +2 \alpha_{n}\left\|\left(u+\gamma f\left(x_{n}\right)\right)-(I+\mu V) W_{n} z_{n}\right\|\left\|y_{n}-p\right\| \\
\leq & \left\|x_{n}-p\right\|^{2}-\left(1-\beta_{n}\right)\left\|\Lambda^{i-1} u_{n}-\Lambda^{i} u_{n}\right\|^{2} \\
& +2 \mu_{i}\left\|\Lambda^{i-1} u_{n}-\Lambda^{i} u_{n}\right\|\left\|B_{i} \Lambda^{i-1} u_{n}-B_{i} p\right\| \\
& +2 \alpha_{n}\left\|\left(u+\gamma f\left(x_{n}\right)\right)-(I+\mu V) W_{n} z_{n}\right\|\left\|y_{n}-p\right\|,
\end{aligned}
$$

which yields

$$
\begin{aligned}
& (1-f)\left\|\Lambda^{i-1} u_{n}-\Lambda^{i} u_{n}\right\|^{2} \\
& \leq\left(1-\beta_{n}\right)\left\|\Lambda^{i-1} u_{n}-\Lambda^{i} u_{n}\right\|^{2} \\
& \leq\left\|x_{n}-p\right\|^{2}-\left\|y_{n}-p\right\|^{2} \\
& \quad+2 \mu_{i}\left\|\Lambda^{i-1} u_{n}-\Lambda^{i} u_{n}\right\|\left\|B_{i} \Lambda^{i-1} u_{n}-B_{i} p\right\| \\
& \quad+2 \alpha_{n}\left\|\left(u+\gamma f\left(x_{n}\right)\right)-(I+\mu V) W_{n} z_{n}\right\| \\
& \quad \times\left\|y_{n}-p\right\|
\end{aligned}
$$

$$
\begin{aligned}
\leq & \left\|x_{n}-y_{n}\left(\left\|x_{n}-p\right\|+\left\|y_{n}-p\right\|\right)+2 \mu_{i}\right\| \\
& \times\left\|\Lambda^{i-1} u_{n}-\Lambda^{i} u_{n}\right\|\left\|B_{i} \Lambda^{i-1} u_{n}-B_{i} p\right\| \\
& +2 \alpha_{n}\left\|\left(u+\gamma f\left(x_{n}\right)\right)-(I+\mu V) W_{n} z_{n}\right\| \\
& \times\left\|y_{n}-p\right\| .
\end{aligned}
$$

From (85), (98), and $\lim _{n \rightarrow \infty} \alpha_{n}=0$, we get

$$
\lim _{n \rightarrow \infty}\left\|\Lambda^{i-1} u_{n}-\Lambda^{i} u_{n}\right\|=0, \quad \forall i \in\{1,2, \ldots, N\} .
$$

From (103) we get

$$
\begin{aligned}
\left\|u_{n}-z_{n}\right\| \\
=\left\|\Lambda^{0} u_{n}-\Lambda^{N} u_{n}\right\| \\
\leq\left\|\Lambda^{0} u_{n}-\Lambda^{1} u_{n}\right\|+\left\|\Lambda^{1} u_{n}-\Lambda^{2} u_{n}\right\| \\
\quad+\cdots+\left\|\Lambda^{N-1} u_{n}-\Lambda^{N} u_{n}\right\| \\
\quad \longrightarrow 0 \quad \text { as } n \longrightarrow \infty .
\end{aligned}
$$

By (94) and (104), we have

$$
\begin{aligned}
\left\|x_{n}-z_{n}\right\| & \leq\left\|x_{n}-u_{n}\right\|+\left\|u_{n}-z_{n}\right\| \\
& \longrightarrow 0 \quad \text { as } n \longrightarrow \infty,
\end{aligned}
$$

together with (85), yields

$$
\begin{aligned}
\left\|y_{n}-z_{n}\right\| & \leq\left\|y_{n}-x_{n}\right\|+\left\|x_{n}-z_{n}\right\| \\
& \longrightarrow 0 \quad \text { as } n \longrightarrow \infty .
\end{aligned}
$$

Note that

$$
\left\|z_{n}-W_{n} z_{n}\right\| \leq\left\|y_{n}-z_{n}\right\|+\left\|x_{n}-W_{n} z_{n}\right\| .
$$

Hence from (83) and (106) we have

$$
\lim _{n \rightarrow \infty}\left\|z_{n}-W_{n} z_{n}\right\|=0 .
$$

Also, observe that

$$
\left\|z_{n}-W z_{n}\right\| \leq\left\|z_{n}-W_{n} z_{n}\right\|+\left\|W_{n} z_{n}-W z_{n}\right\| .
$$

From (108), Remark 8, and the boundedness of $\left\{z_{n}\right\}$ we immediately get

$$
\lim _{n \rightarrow \infty}\left\|z_{n}-W z_{n}\right\|=0
$$

Step 5. We show that $\lim _{\sup _{n \rightarrow \infty}}\left\langle(\gamma f-\mu F) x^{*}, x_{n}-x^{*}\right\rangle \leq 0$, where $x^{*} \in \Omega$ is a unique solution of the VIP:

$$
\left\langle(\gamma f-\mu F) x^{*}, y-x^{*}\right\rangle \leq 0, \quad \forall y \in \Omega .
$$


Indeed, it is clear that $\mu F-\gamma f: H \rightarrow H$ is $(\mu \kappa+\gamma l)$ Lipschitzian. Note that

$$
\begin{aligned}
\mu \eta & \geq \tau \\
& \Longleftrightarrow \mu \eta \geq 1-\sqrt{1-\mu\left(2 \eta-\mu \kappa^{2}\right)} \\
& \Longleftrightarrow \sqrt{1-\mu\left(2 \eta-\mu \kappa^{2}\right)} \geq 1-\mu \eta \\
& \Longleftrightarrow 1-2 \mu \eta+\mu^{2} \kappa^{2} \geq 1-2 \mu \eta+\mu^{2} \eta^{2} \\
& \Longleftrightarrow \kappa^{2} \geq \eta^{2} \\
& \Longleftrightarrow \kappa \geq \eta .
\end{aligned}
$$

It is clear that

$$
\begin{array}{r}
\langle(\mu F-\gamma f) x-(\mu F-\gamma f) y, x-y\rangle \geq(\mu \eta-\gamma l)\|x-y\|^{2} \\
\forall x, y \in H .
\end{array}
$$

Hence by Lemma 12 we deduce from $0 \leq \gamma l<\tau \leq \mu \eta$ that $\mu F-\gamma f$ is $(\mu \eta-\gamma l)$-strongly monotone. In the meantime, it is easy to see that $\mu F-\gamma f$ is $(\mu \kappa+\gamma l)$-Lipschitzian with the constant $\mu \kappa+\gamma l>0$. Thus, there exists a unique solution $x^{*}$ in $\Omega$ to the VIP (111). Equivalently, $P_{\Omega}(I-(\mu F-\gamma f))$ has a unique fixed point $x^{*} \in \Omega$; that is, $x^{*}=P_{\Omega}(I-(\mu F-\gamma f)) x^{*}$.

First, we observe that there exists a subsequence $\left\{x_{n_{i}}\right\}$ of $\left\{x_{n}\right\}$ such that

$$
\begin{aligned}
\limsup _{n \rightarrow \infty}\left\langle(\gamma f-\mu F) x^{*}, x_{n}-x^{*}\right\rangle \\
\quad=\lim _{i \rightarrow \infty}\left\langle(\gamma f-\mu F) x^{*}, x_{n_{i}}-x^{*}\right\rangle .
\end{aligned}
$$

Since $\left\{x_{n_{i}}\right\}$ is bounded, there exists a subsequence $\left\{x_{n_{i_{j}}}\right\}$ of $\left\{x_{n_{i}}\right\}$ which converges weakly to some $w$. Without loss of generality, we may assume that $x_{n_{i}} \rightarrow w$. From (94) and (103)-(105), we have that $u_{n_{i}} \rightarrow w, \Lambda^{m} u_{n_{i}} \rightarrow w$ and $z_{n_{i}} \rightarrow$ $w$, where $m \in\{1,2, \ldots, N\}$. By (110) we have that $\| W z_{n}-$ $z_{n} \| \rightarrow 0$ as $n \rightarrow \infty$. Then, by Lemma 10 we obtain $w \in \operatorname{Fix}(W)=\bigcap_{n=1}^{\infty} \operatorname{Fix}\left(T_{n}\right)$ (due to Lemma 9). Next, we prove that $w \in \bigcap_{m=1}^{N} I\left(B_{m}, R_{m}\right)$. As a matter of fact, since $B_{m}$ is $\eta_{m}$-inverse strongly monotone, $B_{m}$ is a monotone and Lipschitz continuous mapping. It follows from Lemma 17 that $R_{m}+B_{m}$ is maximal monotone. Let $(v, g) \in G\left(R_{m}+B_{m}\right)$; that is, $g-B_{m} v \in R_{m} v$. Again, since $\Lambda^{m} u_{n}=J_{R_{m}, \mu_{m}}(I-$ $\left.\mu_{m} B_{m}\right) \Lambda^{m-1} u_{n}, n \geq 1, m \in\{1,2, \ldots, N\}$, we have

$$
\Lambda^{m-1} u_{n}-\mu_{m} B_{m} \Lambda^{m-1} u_{n} \in\left(I+\mu_{m} R_{m}\right) \Lambda^{m} u_{n}
$$

that is,

$$
\frac{1}{\mu_{m}}\left(\Lambda^{m-1} u_{n}-\Lambda^{m} u_{n}-\mu_{m} B_{m} \Lambda^{m-1} u_{n}\right) \in R_{m} \Lambda^{m} u_{n}
$$

In terms of the monotonicity of $R_{m}$, we get

$$
\begin{aligned}
& \left\langle v-\Lambda^{m} u_{n}, g-B_{m} v\right. \\
& \left.-\frac{1}{\mu_{m}}\left(\Lambda^{m-1} u_{n}-\Lambda^{m} u_{n}-\mu_{m} B_{m} \Lambda^{m-1} u_{n}\right)\right\rangle \geq 0,
\end{aligned}
$$

and hence

$$
\begin{aligned}
& \left\langle v-\Lambda^{m} u_{n}, g\right\rangle \\
& \geq\left\langle v-\Lambda^{m} u_{n}, B_{m} v\right. \\
& \left.\quad \quad+\frac{1}{\mu_{m}}\left(\Lambda^{m-1} u_{n}-\Lambda^{m} u_{n}-\mu_{m} B_{m} \Lambda^{m-1} u_{n}\right)\right\rangle \\
& =\left\langle v-\Lambda^{m} u_{n}, B_{m} v-B_{m} \Lambda^{m} u_{n}+B_{m} \Lambda^{m} u_{n}\right. \\
& \left.\quad-B_{m} \Lambda^{m-1} u_{n}+\frac{1}{\mu_{m}}\left(\Lambda^{m-1} u_{n}-\Lambda^{m} u_{n}\right)\right\rangle \\
& \geq\left\langle v-\Lambda^{m} u_{n}, B_{m} \Lambda^{m} u_{n}-B_{m} \Lambda^{m-1} u_{n}\right\rangle \\
& \quad \quad\left\langle v-\Lambda^{m} u_{n}, \frac{1}{\mu_{m}}\left(\Lambda^{m-1} u_{n}-\Lambda^{m} u_{n}\right)\right\rangle .
\end{aligned}
$$

In particular,

$$
\begin{aligned}
\left\langle v-\Lambda^{m} u_{n_{i}}, g\right\rangle \geq\langle & \left.v-\Lambda^{m} u_{n_{i}}, B_{m} \Lambda^{m} u_{n_{i}}-B_{m} \Lambda^{m-1} u_{n_{i}}\right\rangle \\
& +\left\langle v-\Lambda^{m} u_{n_{i}}, \frac{1}{\mu_{m}}\left(\Lambda^{m-1} u_{n_{i}}-\Lambda^{m} u_{n_{i}}\right)\right\rangle .
\end{aligned}
$$

Since $\left\|\Lambda^{m} u_{n}-\Lambda^{m-1} u_{n}\right\| \rightarrow 0$ (due to (103)) and $\| B_{m} \Lambda^{m} u_{n}-$ $B_{m} \Lambda^{m-1} u_{n} \| \rightarrow 0$ (due to the Lipschitz continuity of $B_{m}$ ), we conclude from $\Lambda^{m} u_{n_{i}} \rightarrow w$ and $\mu_{m} \in\left(0,2 \eta_{m}\right), m \in$ $\{1,2, \ldots, N\}$ that

$$
\lim _{i \rightarrow \infty}\left\langle v-\Lambda^{m} u_{n_{i}}, g\right\rangle=\langle v-w, g\rangle \geq 0 .
$$

It follows from the maximal monotonicity of $B_{m}+R_{m}$ that $0 \in\left(R_{m}+B_{m}\right) w$; that is, $w \in I\left(B_{m}, R_{m}\right)$. Therefore, $w \in$ $\bigcap_{m=1}^{N} I\left(B_{m}, R_{m}\right)$.

Next, we show that $w \in \operatorname{GMEP}(\Theta, \varphi, A)$. In fact, from $z_{n}=S_{r_{n}}^{(\Theta, \varphi)}\left(I-r_{n} A\right) x_{n}$, we know that

$$
\begin{aligned}
& \Theta\left(u_{n}, y\right)+\varphi(y)-\varphi\left(u_{n}\right)+\left\langle A x_{n}, y-u_{n}\right\rangle \\
& +\frac{1}{r_{n}}\left\langle K^{\prime}\left(u_{n}\right)-K^{\prime}\left(x_{n}\right), y-u_{n}\right\rangle \geq 0, \quad \forall y \in C .
\end{aligned}
$$

From $(\mathrm{H} 2)$ it follows that

$$
\begin{aligned}
& \varphi(y)-\varphi\left(u_{n}\right)+\left\langle A x_{n}, y-u_{n}\right\rangle \\
& +\frac{1}{r_{n}}\left\langle K^{\prime}\left(u_{n}\right)-K^{\prime}\left(x_{n}\right), y-u_{n}\right\rangle \\
& \geq \Theta\left(y, u_{n}\right), \quad \forall y \in C .
\end{aligned}
$$


Replacing $n$ by $n_{i}$, we have

$$
\begin{aligned}
& \varphi(y)-\varphi\left(u_{n_{i}}\right)+\left\langle A x_{n_{i}}, y-u_{n_{i}}\right\rangle \\
& +\left\langle\frac{K^{\prime}\left(u_{n_{i}}\right)-K^{\prime}\left(x_{n_{i}}\right)}{r_{n_{i}}}, y-u_{n_{i}}\right\rangle \\
& \geq \Theta\left(y, u_{n_{i}}\right), \quad \forall y \in C .
\end{aligned}
$$

Put $u_{t}=t y+(1-t) w$ for all $t \in(0,1]$ and $y \in C$. Then, from (123) we have

$$
\begin{aligned}
\left\langle u_{t}\right. & \left.-u_{n_{i}}, A u_{t}\right\rangle \\
\geq & \left\langle u_{t}-u_{n_{i}}, A u_{t}\right\rangle-\varphi\left(u_{t}\right)+\varphi\left(u_{n_{i}}\right)-\left\langle u_{t}-u_{n_{i}}, A x_{n_{i}}\right\rangle \\
& -\left\langle\frac{K^{\prime}\left(u_{n_{i}}\right)-K^{\prime}\left(x_{n_{i}}\right)}{r_{n_{i}}}, u_{t}-u_{n_{i}}\right\rangle+\Theta\left(u_{t}, u_{n_{i}}\right) \\
\geq & \left\langle u_{t}-u_{n_{i}}, A u_{t}-A u_{n_{i}}\right\rangle+\left\langle u_{t}-u_{n_{i}}, A u_{n_{i}}-A x_{n_{i}}\right\rangle \\
& -\varphi\left(u_{t}\right)+\varphi\left(u_{n_{i}}\right) \\
& -\left\langle\frac{K^{\prime}\left(u_{n_{i}}\right)-K^{\prime}\left(x_{n_{i}}\right)}{r_{n_{i}}}, u_{t}-u_{n_{i}}\right\rangle+\Theta\left(u_{t}, u_{n_{i}}\right) .
\end{aligned}
$$

Since $\left\|u_{n_{i}}-x_{n_{i}}\right\| \rightarrow 0$ as $i \rightarrow \infty$, we deduce from the Lipschitz continuity of $A$ and $K^{\prime}$ that $\left\|A u_{n_{i}}-A x_{n_{i}}\right\| \rightarrow 0$ and $\left\|K^{\prime}\left(u_{n_{i}}\right)-K^{\prime}\left(x_{n_{i}}\right)\right\| \rightarrow 0$ as $i \rightarrow \infty$. Further, from the monotonicity of $A$, we have $\left\langle u_{t}-u_{n_{i}}, A u_{t}-A u_{n_{i}}\right\rangle \geq 0$. So, from (H4), the weakly lower semicontinuity of $\varphi,\left(\left(K^{\prime}\left(u_{n_{i}}\right)-\right.\right.$ $\left.\left.K^{\prime}\left(x_{n_{i}}\right)\right) / r_{n_{i}}\right) \rightarrow 0$ and $u_{n_{i}} \rightarrow w$, we have

$$
\left\langle u_{t}-w, A u_{t}\right\rangle \geq-\varphi\left(u_{t}\right)+\varphi(w)+\Theta\left(u_{t}, w\right), \quad \text { as } i \longrightarrow \infty .
$$

From (H1), (H4), and (125) we also have

$$
\begin{aligned}
0= & \Theta\left(u_{t}, u_{t}\right)+\varphi\left(u_{t}\right)-\varphi\left(u_{t}\right) \\
\leq & t \Theta\left(u_{t}, y\right)+(1-t) \Theta\left(u_{t}, w\right)+t \varphi(y) \\
& +(1-t) \varphi(w)-\varphi\left(u_{t}\right) \\
= & t\left[\Theta\left(u_{t}, y\right)+\varphi(y)-\varphi\left(u_{t}\right)\right] \\
& \quad+(1-t)\left[\Theta\left(u_{t}, w\right)+\varphi(w)-\varphi(w)-\varphi\left(u_{t}\right)\right] \\
\leq & t\left[\Theta\left(u_{t}, y\right)+\varphi(y)-\varphi\left(u_{t}\right)\right]+(1-t)\left\langle u_{t}-w, A u_{t}\right\rangle \\
= & t\left[\Theta\left(u_{t}, y\right)+\varphi(y)-\varphi\left(u_{t}\right)\right]+(1-t) t\left\langle y-w, A u_{t}\right\rangle,
\end{aligned}
$$

and hence

$$
0 \leq \Theta\left(u_{t}, y\right)+\varphi(y)-\varphi\left(u_{t}\right)+(1-t)\left\langle y-w, A u_{t}\right\rangle .
$$

Letting $t \rightarrow 0$, we have, for each $y \in C$,

$$
0 \leq \Theta(w, y)+\varphi(y)-\varphi(w)+\langle A w, y-w\rangle
$$

This implies that $w \in \operatorname{GMEP}(\Theta, \varphi, A)$. Therefore, $w \in$ $\bigcap_{n=1}^{\infty} \operatorname{Fix}\left(T_{n}\right) \cap \operatorname{GMEP}(\Theta, \varphi, A) \cap \bigcap_{i=1}^{N} I\left(B_{i}, R_{i}\right):=\Omega$. This shows that $\omega_{w}\left(x_{n}\right) \subset \Omega$. Consequently, from (111) and (114) we have

$$
\begin{array}{r}
\limsup _{n \rightarrow \infty}\left\langle(\gamma f-\mu F) x^{*}, x_{n}-x^{*}\right\rangle \\
=\left\langle(\gamma f-\mu F) x^{*}, w-x^{*}\right\rangle \leq 0 .
\end{array}
$$

Step 6. Finally, we show that $x_{n} \rightarrow x^{*} \in \Omega$, where $x^{*}=$ $P_{\Omega}(I-(\mu F-\gamma f)) x^{*}$.

Indeed, in terms of Lemma 4 we have

$$
\begin{aligned}
& \left\|y_{n}-x^{*}\right\|^{2} \\
& =\| \alpha_{n}\left(u+\gamma f\left(x_{n}\right)-\bar{V} x^{*}\right)+\beta_{n}\left(x_{n}-x^{*}\right) \\
& +\left(\left(1-\beta_{n}\right) I-\alpha_{n} \bar{V}\right)\left(W_{n} z_{n}-x^{*}\right) \|^{2} \\
& \leq\left\|\beta_{n}\left(x_{n}-x^{*}\right)+\left(\left(1-\beta_{n}\right) I-\alpha_{n} \bar{V}\right)\left(W_{n} z_{n}-x^{*}\right)\right\|^{2} \\
& +2 \alpha_{n}\left\langle u+\gamma f\left(x_{n}\right)-\bar{V} x^{*}, y_{n}-x^{*}\right\rangle \\
& \leq\left[\left\|\left(\left(1-\beta_{n}\right) I-\alpha_{n} \bar{V}\right)\left(W_{n} z_{n}-x^{*}\right)\right\|\right. \\
& \left.+\beta_{n}\left\|x_{n}-x^{*}\right\|\right]^{2}+2 \alpha_{n}\left\|u+\gamma f\left(x_{n}\right)-\bar{V} x^{*}\right\|\left\|y_{n}-x^{*}\right\| \\
& \leq\left[\left(1-\beta_{n}-\alpha_{n}(1+\mu) \bar{\gamma}\right)\left\|W_{n} z_{n}-x^{*}\right\|+\beta_{n}\left\|x_{n}-x^{*}\right\|\right]^{2} \\
& +2 \alpha_{n}\left\|u+\gamma f\left(x_{n}\right)-\bar{V} x^{*}\right\|\left\|y_{n}-x^{*}\right\| \\
& \leq\left[\left(1-\beta_{n}-\alpha_{n}(1+\mu) \bar{\gamma}\right)\left\|z_{n}-x^{*}\right\|+\beta_{n}\left\|x_{n}-x^{*}\right\|\right]^{2} \\
& +2 \alpha_{n}\left\|u+\gamma f\left(x_{n}\right)-\bar{V} x^{*}\right\|\left\|y_{n}-x^{*}\right\| \\
& \leq\left[\left(1-\beta_{n}-\alpha_{n}(1+\mu) \bar{\gamma}\right)\left\|x_{n}-x^{*}\right\|+\beta_{n}\left\|x_{n}-x^{*}\right\|\right]^{2} \\
& +2 \alpha_{n}\left\|u+\gamma f\left(x_{n}\right)-\bar{V} x^{*}\right\|\left\|y_{n}-x^{*}\right\| \\
& =\left(1-\alpha_{n}(1+\mu) \bar{\gamma}\right)^{2}\left\|x_{n}-x^{*}\right\|^{2} \\
& +2 \alpha_{n}\left\|u+\gamma f\left(x_{n}\right)-\bar{V} x^{*}\right\|\left\|y_{n}-x^{*}\right\| \\
& \leq\left\|x_{n}-x^{*}\right\|^{2}+2 \alpha_{n}\left\|u+\gamma f\left(x_{n}\right)-\bar{V} x^{*}\right\|\left\|y_{n}-x^{*}\right\| \text {. }
\end{aligned}
$$

Utilizing Lemmas 4 and 13, we conclude from (130) that

$$
\begin{aligned}
& \left\|x_{n+1}-x^{*}\right\|^{2} \\
& =\| \sigma_{n} \gamma\left(f\left(y_{n}\right)-f\left(x^{*}\right)\right)+\left(I-\sigma_{n} \mu F\right) W_{n} y_{n} \\
& \quad-\left(I-\sigma_{n} \mu F\right) W_{n} x^{*}+\sigma_{n}\left(\gamma f\left(x^{*}\right)-\mu F x^{*}\right) \|^{2} \\
& \leq \| \sigma_{n} \gamma\left(f\left(y_{n}\right)-f\left(x^{*}\right)\right)+\left(I-\sigma_{n} \mu F\right) W_{n} y_{n} \\
& \quad-\left(I-\sigma_{n} \mu F\right) W_{n} x^{*} \|^{2} \\
& +2 \sigma_{n}\left\langle\gamma f\left(x^{*}\right)-\mu F x^{*}, x_{n+1}-x^{*}\right\rangle
\end{aligned}
$$




$$
\begin{aligned}
\leq & {\left[\sigma_{n} \gamma\left\|f\left(y_{n}\right)-f\left(x^{*}\right)\right\|\right.} \\
& \left.+\left\|\left(I-\sigma_{n} \mu F\right) W_{n} y_{n}-\left(I-\sigma_{n} \mu F\right) W_{n} x^{*}\right\|\right]^{2} \\
& +2 \sigma_{n}\left\langle\gamma f\left(x^{*}\right)-\mu F x^{*}, x_{n+1}-x^{*}\right\rangle \\
\leq & {\left[\sigma_{n} \gamma l\left\|y_{n}-x^{*}\right\|+\left(1-\sigma_{n} \tau\right)\left\|y_{n}-x^{*}\right\|\right]^{2} } \\
& +2 \sigma_{n}\left\langle\gamma f\left(x^{*}\right)-\mu F x^{*}, x_{n+1}-x^{*}\right\rangle \\
= & \left(1-\sigma_{n}(\tau-\gamma l)\right)^{2}\left\|y_{n}-x^{*}\right\|^{2} \\
& +2 \sigma_{n}\left\langle\gamma f\left(x^{*}\right)-\mu F x^{*}, x_{n+1}-x^{*}\right\rangle \\
\leq & \left(1-\sigma_{n}(\tau-\gamma l)\right) \\
& \times \\
+ & {\left[\left\|x_{n}-x^{*}\right\|^{2}+2 \alpha_{n}\left\|u+\gamma f\left(x_{n}\right)-\bar{V} x^{*}\right\|\left\|y_{n}-x^{*}\right\|\right] } \\
& +2 \sigma_{n}\left\langle\gamma f\left(x^{*}\right)-\mu F x^{*}, x_{n+1}-x^{*}\right\rangle \\
\leq & \left(1-\sigma_{n}(\tau-\gamma l)\right)\left\|x_{n}-x^{*}\right\|^{2} \\
& +2 \alpha_{n}\left\|u+\gamma f\left(x_{n}\right)-\bar{V} x^{*}\right\|\left\|y_{n}-x^{*}\right\| \\
& +2 \sigma_{n}\left\langle\gamma f\left(x^{*}\right)-\mu F x^{*}, x_{n+1}-x^{*}\right\rangle \\
= & \left(1-\sigma_{n}(\tau-\gamma l)\right)\left\|x_{n}-x^{*}\right\|^{2} \\
+ & \sigma_{n}(\tau-\gamma l) \cdot \frac{\alpha_{n}}{\tau-\gamma l}\left\|u+\gamma f\left(x_{n}\right)-\bar{V} x^{*}\right\|
\end{aligned}
$$

Note that $0 \leq \gamma l<\tau$. Hence, $\sum_{n=1}^{\infty} \sigma_{n}=\infty$ leads to $\sum_{n=1}^{\infty}$ $\sigma_{n}(\tau-\gamma l)=\infty$. In addition, since $\lim _{n \rightarrow \infty} \sigma_{n}=0$ and $\lim _{n \rightarrow \infty}\left(\alpha_{n} / \sigma_{n}\right)=0$, we get from (129)

$$
\begin{array}{r}
\limsup _{n \rightarrow \infty} \frac{2}{\tau-\gamma l}\left[\frac{\alpha_{n}}{\sigma_{n}}\left\|u+\gamma f\left(x_{n}\right)-\bar{V} x^{*}\right\|\left\|y_{n}-x^{*}\right\|\right. \\
\left.+\left\langle\gamma f\left(x^{*}\right)-\mu F x^{*}, x_{n+1}-x^{*}\right\rangle\right] \leq 0 .
\end{array}
$$

Applying Lemma 19 to (131), we have that $x_{n} \rightarrow x^{*}$ as $n \rightarrow$ $\infty$. This completes the proof.

Corollary 21. Let $C$ be a nonempty closed convex subset of a real Hilbert space $H$. Let $N$ be an integer. Let $\Theta$ be a bifunction from $\mathrm{C} \times \mathrm{C}$ to $\mathbf{R}$ satisfying $(\mathrm{H} 1)-(\mathrm{H} 4)$ and $\varphi: C \rightarrow \mathbf{R}$ be a lower semicontinuous and convex functional. Let $R_{i}: C \rightarrow 2^{H}$ be a maximal monotone mapping and let $A: H \rightarrow H$ and $B_{i}: C \rightarrow H$ be $\zeta$-inverse strongly monotone and $\eta_{i^{-}}$ inverse strongly monotone, respectively, where $i \in\{1,2, \ldots, N\}$. Let $\left\{T_{n}\right\}_{n=1}^{\infty}$ be a sequence of nonexpansive self-mappings on $H$ and $\left\{\lambda_{n}\right\}$ be a sequence in $(0, b]$ for some $b \in(0,1)$. Let $V$ be a $\bar{\gamma}$-strongly positive bounded linear operator. Given $u \in H$ and $\mu>0$, let $F x=((1 / \mu) I+V) x-(1 / \mu) u$. Suppose $0<\mu((1 / \mu)+\|V\|)^{2}<2((1 / \mu)+\bar{\gamma})$ and $\tau=$ $1-\sqrt{1-\mu\left(2((1 / \mu)+\bar{\gamma})-\mu((1 / \mu)+\|V\|)^{2}\right)}$. Let $f: H \rightarrow H$ be an l-Lipschitzian mapping with $0 \leq \gamma l<\min \{\tau,(1+\mu) \bar{\gamma}\}$. Let $W_{n}$ be the $W$-mapping defined by (15). Assume that $\Omega:=$ $\bigcap_{n=1}^{\infty} \operatorname{Fix}\left(T_{n}\right) \cap \operatorname{GMEP}(\Theta, \varphi, A) \cap \bigcap_{i=1}^{N} I\left(B_{i}, R_{i}\right)$ is nonempty. Let $\left\{\alpha_{n}\right\},\left\{\beta_{n}\right\}$, and $\left\{\sigma_{n}\right\}$ be three sequences in $(0,1)$. Assume that the conditions (i)-(v) of Theorem 20 hold. If $S_{r}^{(\Theta, \varphi)}$ is firmly nonexpansive, then for a given arbitrary $x_{1} \in H$, the sequence $\left\{x_{n}\right\}$ generated iteratively by

$$
\begin{gathered}
u_{n}=S_{r_{n}}^{(\Theta, \varphi)}\left(I-r_{n} A\right) x_{n}, \\
z_{n}=J_{R_{N}, \mu_{N}}\left(I-\mu_{N} B_{N}\right) J_{R_{N-1}, \mu_{N-1}}\left(I-\mu_{N-1} B_{N-1}\right) \\
\cdots J_{R_{1}, \mu_{1}}\left(I-\mu_{1} B_{1}\right) u_{n}, \\
y_{n}=\alpha_{n}\left(u+\gamma f\left(x_{n}\right)\right)+\beta_{n} x_{n} \\
+\left(\left(1-\beta_{n}\right) I-\alpha_{n}(I+\mu V)\right) W_{n} z_{n}, \\
x_{n+1}=\sigma_{n}\left(u+\gamma f\left(y_{n}\right)\right)+\left(I-\sigma_{n}(I+\mu V)\right) W_{n} y_{n}, \\
\quad \forall n \geq 1,
\end{gathered}
$$

converges strongly to $x^{*} \in \Omega$, which solves the following optimization problem:

$$
\min _{x \in \Omega} \frac{\mu}{2}\langle V x, x\rangle+\frac{1}{2}\|x-u\|^{2}-h(x),
$$

where $h: H \rightarrow \mathbf{R}$ is the potential function of $\gamma f$.

Proof. Given $u \in H$ and $\mu>0$, let $F x=((1 / \mu) I+$ $V) x-(1 / \mu) u$. Then $F: H \rightarrow H$ is a $\kappa$-Lipschitzian and $\eta$-strongly monotone operator with positive constants $\kappa=(1 / \mu)+\|V\|$ and $\eta=(1 / \mu)+\bar{\gamma}$. Suppose $0<\mu<$ $\left(2 \eta / \kappa^{2}\right)=(2((1 / \mu)+\bar{\gamma})) /\left(((1 / \mu)+\|V\|)^{2}\right)$ and $\tau=1-$ $\sqrt{1-\mu\left(2((1 / \mu)+\bar{\gamma})-\mu((1 / \mu)+\|V\|)^{2}\right)}$. In this case, it is easy from (56) to see that

$$
\begin{aligned}
x_{n+1} & =\sigma_{n} \gamma f\left(y_{n}\right)+\left(I-\sigma_{n} \mu F\right) W_{n} y_{n} \\
& =\sigma_{n} \gamma f\left(y_{n}\right)+\left(I-\sigma_{n}(I+\mu V)\right) W_{n} y_{n}+\sigma_{n} u \\
& =\sigma_{n}\left(u+\gamma f\left(y_{n}\right)\right)+\left(I-\sigma_{n}(I+\mu V)\right) W_{n} y_{n} .
\end{aligned}
$$

Then, for $0 \leq \gamma l<\min \{\tau,(1+\mu) \bar{\gamma}\}$, all conditions of Theorem 20 are satisfied. Therefore, utilizing Theorem 20 we infer that $\left\{x_{n}\right\}$ converges strongly to $x^{*} \in \Omega$, where $x^{*}=$ $P_{\Omega}(I-(\mu F-\gamma f)) x^{*}$ is a unique solution of the VIP:

$$
\left\langle(\gamma f-\mu F) x^{*}, y-x^{*}\right\rangle \leq 0, \quad \forall y \in \Omega .
$$

Utilizing Lemma 18, we know that $x^{*}$ solves the following optimization problem:

$$
\min _{x \in \Omega} \frac{\mu}{2}\langle V x, x\rangle+\frac{1}{2}\|x-u\|^{2}-h(x),
$$

where $h: H \rightarrow \mathbf{R}$ is the potential function of $\gamma f$. 
Corollary 22. Let $C$ be a nonempty closed convex subset of a real Hilbert space $H$. Let $\Theta$ be a bifunction from $C \times C$ to $\mathbf{R}$ satisfying (H1)-(H4) and $\varphi: C \rightarrow \mathbf{R}$ be a lower semicontinuous and convex functional. Let $R_{i}: C \rightarrow 2^{H}$ be a maximal monotone mapping and let $A: H \rightarrow H$ and $B_{i}: C \rightarrow H$ be $\zeta$-inverse strongly monotone and $\eta_{i}$-inverse strongly monotone, respectively, for $i=1,2$. Let $\left\{T_{n}\right\}_{n=1}^{\infty}$ be a sequence of nonexpansive self-mappings on $H$ and $\left\{\lambda_{n}\right\}$ be a sequence in $(0, b]$ for some $b \in(0,1)$. Let $F: H \rightarrow H$ be a $\kappa$-Lipschitzian and $\eta$-strongly monotone operator with positive constants $\kappa, \eta>0$. Let $0<\mu<\left(2 \eta / \kappa^{2}\right)$ and $\tau=1-\sqrt{1-\mu\left(2 \eta-\mu \kappa^{2}\right)}$. Let $f: H \rightarrow H$ be an l-Lipschitzian mapping with $0 \leq \gamma l<\tau$. Let $W_{n}$ be the $W$-mapping defined by (15) and $V$ be a $\bar{\gamma}$-strongly positive bounded linear operator with $\gamma l<(1+\mu) \bar{\gamma}$. Assume that $\Omega:=\bigcap_{n=1}^{\infty} \operatorname{Fix}\left(T_{n}\right) \cap \operatorname{GMEP}(\Theta, \varphi, A) \cap I\left(B_{2}, R_{2}\right) \cap I\left(B_{1}, R_{1}\right)$ is nonempty. Let $\left\{\alpha_{n}\right\},\left\{\beta_{n}\right\}$, and $\left\{\sigma_{n}\right\}$ be three sequences in $(0,1)$. Assume that the conditions (i)-( $v)$ of Theorem 20 hold. If $S_{r}^{(\Theta, \varphi)}$ is firmly nonexpansive, then for a given arbitrary $x_{1} \in H$, the sequence $\left\{x_{n}\right\}$ generated iteratively by

$$
\begin{gathered}
u_{n}=S_{r_{n}}^{(\Theta, \varphi)}\left(I-r_{n} A\right) x_{n}, \\
z_{n}=J_{R_{2}, \mu_{2}}\left(I-\mu_{2} B_{2}\right) J_{R_{1}, \mu_{1}}\left(I-\mu_{1} B_{1}\right) u_{n}, \\
y_{n}=\alpha_{n}\left(u+\gamma f\left(x_{n}\right)\right)+\beta_{n} x_{n} \\
+\left(\left(1-\beta_{n}\right) I-\alpha_{n}(I+\mu V)\right) W_{n} z_{n}, \\
x_{n+1}=\sigma_{n} \gamma f\left(y_{n}\right)+\left(I-\sigma_{n} \mu F\right) W_{n} y_{n}, \quad \forall n \geq 1,
\end{gathered}
$$

converges strongly to $x^{*} \in \Omega$, where $x^{*}=P_{\Omega}(I-(\mu F-\gamma f)) x^{*}$ is a unique solution of the VIP:

$$
\left\langle(\gamma f-\mu F) x^{*}, y-x^{*}\right\rangle \leq 0, \quad \forall y \in \Omega
$$

Corollary 23. Let $C$ be a nonempty closed convex subset of a real Hilbert space $H$. Let $N$ be an integer. Let $\Theta$ be a bifunction from $\mathrm{C} \times \mathrm{C}$ to $\mathbf{R}$ satisfying $(\mathrm{H} 1)-(\mathrm{H} 4)$ and $\varphi: C \rightarrow \mathbf{R}$ be a lower semicontinuous and convex functional. Let $R_{i}: C \rightarrow 2^{H}$ be a maximal monotone mapping and let $B_{i}: C \rightarrow H$ be $\eta_{i}{ }^{-}$ inverse strongly monotone, respectively, where $i \in\{1,2, \ldots, N\}$. Let $\left\{T_{n}\right\}_{n=1}^{\infty}$ be a sequence of nonexpansive self-mappings on $H$ and $\left\{\lambda_{n}\right\}$ be a sequence in $(0, b]$ for some $b \in(0,1)$. Let $F$ : $H \rightarrow H$ be $a \kappa$-Lipschitzian and $\eta$-strongly monotone operator with positive constants $\kappa, \eta>0$. Let $0<\mu<\left(2 \eta / \kappa^{2}\right)$ and $\tau=1-\sqrt{1-\mu\left(2 \eta-\mu \kappa^{2}\right)}$. Let $f: H \rightarrow H$ be an l-Lipschitzian mapping with $0 \leq \gamma l<\tau$. Let $W_{n}$ be the $W$-mapping defined by (15) and $V$ be a $\bar{\gamma}$-strongly positive bounded linear operator with $\gamma l<(1+\mu) \bar{\gamma}$. Assume that $\Omega:=\bigcap_{n=1}^{\infty} \operatorname{Fix}\left(T_{n}\right) \cap$ $\operatorname{MEP}(\Theta, \varphi) \cap \bigcap_{i=1}^{N} I\left(B_{i}, R_{i}\right)$ is nonempty. Let $\left\{\alpha_{n}\right\},\left\{\beta_{n}\right\}$, and $\left\{\sigma_{n}\right\}$ be three sequences in $(0,1)$. Assume that the conditions (i)-(v) of Theorem 20 hold, where $\left\{r_{n}\right\}$ is a bounded sequence such that $0<\liminf _{n \rightarrow \infty} r_{n} \leq \limsup _{n \rightarrow \infty} r_{n}<2 \zeta$ for some $\zeta>0$. If $S_{r}^{(\Theta, \varphi)}$ is firmly nonexpansive, then for a given arbitrary $x_{1} \in H$, the sequence $\left\{x_{n}\right\}$ generated iteratively by

$$
\begin{aligned}
& \Theta\left(u_{n}, y\right)+\varphi(y)-\varphi\left(u_{n}\right) \\
& +\frac{1}{r_{n}}\left\langle k^{\prime}\left(u_{n}\right)-k^{\prime}\left(x_{n}\right), y-u_{n}\right\rangle \geq 0, \quad \forall y \in C, \\
& z_{n}=J_{R_{N}, \mu_{N}}\left(I-\mu_{N} B_{N}\right) J_{R_{N-1}, \mu_{N-1}}\left(I-\mu_{N-1} B_{N-1}\right) \\
& \quad \cdots J_{R_{1}, \mu_{1}}\left(I-\mu_{1} B_{1}\right) u_{n}, \\
& y_{n}=\alpha_{n}\left(u+\gamma f\left(x_{n}\right)\right)+\beta_{n} x_{n} \\
& \quad+\left(\left(1-\beta_{n}\right) I-\alpha_{n}(I+\mu V)\right) W_{n} z_{n}, \\
& x_{n+1}=\sigma_{n} \gamma f\left(y_{n}\right)+\left(I-\sigma_{n} \mu F\right) W_{n} y_{n}, \quad \forall n \geq 1,
\end{aligned}
$$

converges strongly to $x^{*} \in \Omega$, where $x^{*}=P_{\Omega}(I-(\mu F-\gamma f)) x^{*}$ is a unique solution of the VIP:

$$
\left\langle(\gamma f-\mu F) x^{*}, y-x^{*}\right\rangle \leq 0, \quad \forall y \in \Omega .
$$

Proof. In Theorem 20, for all $n \geq 1, u_{n}=S_{r_{n}}^{(\Theta, \varphi)}\left(I-r_{n} A\right) x_{n}$ is equivalent to

$$
\begin{aligned}
& \Theta\left(u_{n}, y\right)+\varphi(y)-\varphi\left(u_{n}\right)+\left\langle A x_{n}, y-u_{n}\right\rangle \\
& \quad+\frac{1}{r_{n}}\left\langle k^{\prime}\left(u_{n}\right)-k^{\prime}\left(x_{n}\right), y-u_{n}\right\rangle \geq 0, \quad \forall y \in C .
\end{aligned}
$$

Put $A \equiv 0$. Then it follows that

$$
\begin{aligned}
& \Theta\left(u_{n}, y\right)+\varphi(y)-\varphi\left(u_{n}\right) \\
& +\frac{1}{r_{n}}\left\langle k^{\prime}\left(u_{n}\right)-k^{\prime}\left(x_{n}\right), y-u_{n}\right\rangle \geq 0, \quad \forall y \in C .
\end{aligned}
$$

Observe that for all $\zeta \in(0, \infty)$

$$
\langle A x-A y, x-y\rangle \geq \zeta\|A x-A y\|^{2}, \quad \forall x, y \in H .
$$

So, whenever $0<\liminf _{n \rightarrow \infty} r_{n} \leq \limsup _{n \rightarrow \infty} r_{n}<2 \zeta$ for some $\zeta \in(0, \infty)$, we obtain the desired result by using Theorem 20.

Let $T: H \rightarrow H$ be a $k$-strictly pseudocontractive mapping. For recent convergence result for strictly pseudocontractive mappings, we refer to [44]. Putting $A=I-T$, we know that for all $x, y \in H$

$$
\|(I-A) x-(I-A) y\|^{2} \leq\|x-y\|^{2}+k\|A x-A y\|^{2} .
$$

Note that

$$
\begin{aligned}
& \|(I-A) x-(I-A) y\|^{2} \\
& \quad=\|x-y\|^{2}+\|A x-A y\|^{2}-2\langle A x-A y, x-y\rangle .
\end{aligned}
$$

Hence, we have for all $x, y \in H$

$$
\langle A x-A y, x-y\rangle \geq \frac{1-k}{2}\|A x-A y\|^{2} .
$$

Consequently, if $T: H \rightarrow H$ is a $k$-strictly pseudocontractive mapping, then the mapping $A=I-T$ is $(1-k) / 2$-inverse strongly monotone. 
Corollary 24. Let $C$ be a nonempty closed convex subset of a real Hilbert space $H$. Let $N$ be an integer. Let $\Theta$ be a bifunction from $\mathrm{C} \times \mathrm{C}$ to $\mathbf{R}$ satisfying $(\mathrm{H} 1)-(\mathrm{H} 4)$ and $\varphi: C \rightarrow \mathbf{R}$ be a lower semicontinuous and convex functional. Let $R_{i}: C \rightarrow 2^{H}$ be a maximal monotone mapping and let $T: H \rightarrow H$ and $B_{i}: C \rightarrow H$ be a $k$-strictly pseudocontractive mapping and an $\eta_{i}$-inverse strongly monotone mapping, respectively, where $i \in\{1,2, \ldots, N\}$. Let $\left\{T_{n}\right\}_{n=1}^{\infty}$ be a sequence of nonexpansive self-mappings on $H$ and $\left\{\lambda_{n}\right\}$ be a sequence in $(0, b]$ for some $b \in(0,1)$. Let $F: H \rightarrow H$ be a $\kappa$-Lipschitzian and $\eta$-strongly monotone operator with positive constants $\kappa, \eta>0$. Let $0<$ $\mu<\left(2 \eta / \kappa^{2}\right)$ and $\tau=1-\sqrt{1-\mu\left(2 \eta-\mu \kappa^{2}\right)}$. Let $f: H \rightarrow$ $H$ be an l-Lipschitzian mapping with $0 \leq \gamma l<\tau$. Let $W_{n}$ be the $W$-mapping defined by (15) and $V$ be a $\bar{\gamma}$-strongly positive bounded linear operator with $\gamma l<(1+\mu) \bar{\gamma}$. Assume that $\Omega:=$ $\bigcap_{n=1}^{\infty} \operatorname{Fix}\left(T_{n}\right) \cap \operatorname{GMEP}(\Theta, \varphi, A) \cap \bigcap_{i=1}^{N} I\left(B_{i}, R_{i}\right)$ is nonempty, where $A=I-T$. Let $\left\{\alpha_{n}\right\},\left\{\beta_{n}\right\}$, and $\left\{\sigma_{n}\right\}$ be three sequences in $(0,1)$. Assume that the conditions $(i)-(v)$ of Theorem 20 hold where $\zeta=((1-k) / 2)$. If $S_{r}^{(\Theta, \varphi)}$ is firmly nonexpansive, then for a given arbitrary $x_{1} \in H$, the sequence $\left\{x_{n}\right\}$ generated iteratively by

$$
\begin{gathered}
u_{n}=S_{r_{n}}^{(\Theta, \varphi)}\left(\left(1-r_{n}\right) x_{n}+r_{n} T x_{n}\right), \\
z_{n}=J_{R_{N}, \mu_{N}}\left(I-\mu_{N} B_{N}\right) J_{R_{N-1}, \mu_{N-1}}\left(I-\mu_{N-1} B_{N-1}\right) \\
\cdots J_{R_{1}, \mu_{1}}\left(I-\mu_{1} B_{1}\right) u_{n}, \\
y_{n}=\alpha_{n}\left(u+\gamma f\left(x_{n}\right)\right)+\beta_{n} x_{n} \\
+\left(\left(1-\beta_{n}\right) I-\alpha_{n}(I+\mu V)\right) W_{n} z_{n}, \\
x_{n+1}=\sigma_{n} \gamma f\left(y_{n}\right)+\left(I-\sigma_{n} \mu F\right) W_{n} y_{n}, \quad \forall n \geq 1,
\end{gathered}
$$

converges strongly to $x^{*} \in \Omega$, where $x^{*}=P_{\Omega}(I-(\mu F-\gamma f)) x^{*}$ is a unique solution of the VIP:

$$
\left\langle(\gamma f-\mu F) x^{*}, y-x^{*}\right\rangle \leq 0, \quad \forall y \in \Omega .
$$

Proof. Since $T$ is a $k$-strictly pseudocontractive mapping, the mapping $A=I-T$ is $(1-k) / 2$-inverse strongly monotone. In this case, put $\zeta=(1-k) / 2$. Moreover, we obtain that

$$
\begin{aligned}
u_{n} & =S_{r_{n}}^{(\Theta, \varphi)}\left(I-r_{n} A\right) x_{n} \\
& =S_{r_{n}}^{(\Theta, \varphi)}\left(x_{n}-r_{n}(I-T) x_{n}\right) \\
& =S_{r_{n}}^{(\Theta, \varphi)}\left(\left(1-r_{n}\right) x_{n}+r_{n} T x_{n}\right) .
\end{aligned}
$$

So, from Theorem 20, we obtain the desired result.

Corollary 25. Let $C$ be a nonempty closed convex subset of a real Hilbert space $H$. Let $N$ be an integer. Let $\Theta$ be a bifunction from $\mathrm{C} \times \mathrm{C}$ to $\mathbf{R}$ satisfying $(\mathrm{H} 1)-(\mathrm{H} 4)$ and $\varphi: C \rightarrow \mathbf{R}$ be a lower semicontinuous and convex functional. Let $R_{i}: C \rightarrow 2^{H}$ be a maximal monotone mapping and let $A: H \rightarrow H$ and $B_{i}: C \rightarrow H$ be $\zeta$-inverse strongly monotone and $\eta_{i}$-inverse strongly monotone, respectively, where $i \in\{1,2, \ldots, N\}$. Let $F: H \rightarrow H$ be a $\kappa$-Lipschitzian and $\eta$-strongly monotone operator with positive constants $\kappa, \eta>0$. Let $0<\mu<\left(2 \eta / \kappa^{2}\right)$ and $\tau=1-\sqrt{1-\mu\left(2 \eta-\mu \kappa^{2}\right)}$. Let $f: H \rightarrow H$ be an l-Lipschitzian mapping with $0 \leq \gamma l<\tau$. Let $V$ be a $\bar{\gamma}$ strongly positive bounded linear operator with $\gamma l<(1+\mu) \bar{\gamma}$. Assume that $\Omega:=\operatorname{GMEP}(\Theta, \varphi, A) \cap \bigcap_{i=1}^{N} I\left(B_{i}, R_{i}\right)$ is nonempty. Let $\left\{\alpha_{n}\right\},\left\{\beta_{n}\right\}$, and $\left\{\sigma_{n}\right\}$ be three sequences in $(0,1)$. Assume that conditions (i)-(v) of Theorem 20 hold. Given $x_{1} \in H$ arbitrarily, let the sequence $\left\{x_{n}\right\}$ be generated iteratively by

$$
\begin{gathered}
u_{n}=S_{r_{n}}^{(\Theta, \varphi)}\left(I-r_{n} A\right) x_{n}, \\
z_{n}=J_{R_{N}, \mu_{N}}\left(I-\mu_{N} B_{N}\right) J_{R_{N-1}, \mu_{N-1}}\left(I-\mu_{N-1} B_{N-1}\right) \\
\cdots J_{R_{1}, \mu_{1}}\left(I-\mu_{1} B_{1}\right) u_{n}, \\
y_{n}=\alpha_{n}\left(u+\gamma f\left(x_{n}\right)\right)+\beta_{n} x_{n} \\
+\left(\left(1-\beta_{n}\right) I-\alpha_{n}(I+\mu V)\right) z_{n}, \\
x_{n+1}=\sigma_{n} \gamma f\left(y_{n}\right)+\left(I-\sigma_{n} \mu F\right) y_{n}, \quad \forall n \geq 1 .
\end{gathered}
$$

If $S_{r}^{(\Theta, \varphi)}$ is firmly nonexpansive, then $\left\{x_{n}\right\}$ converges strongly to $x^{*} \in \Omega$, where $x^{*}=P_{\Omega}(I-(\mu F-\gamma f)) x^{*}$ is a unique solution of the VIP:

$$
\left\langle(\gamma f-\mu F) x^{*}, y-x^{*}\right\rangle \leq 0, \quad \forall y \in \Omega \text {. }
$$

Proof. Put $T_{n} x=x$ for all integers $n \geq 1$ and all $x \in H$. Then, the desired result follows from Theorem 20.

Corollary 26. Let $C$ be a nonempty closed convex subset of a real Hilbert space $H$. Let $N$ be an integer. Let $R_{i}: C \rightarrow$ $2^{H}$ be a maximal monotone mapping and let $B_{i}: C \rightarrow H$ be $\eta_{i}$-inverse strongly monotone, where $i \in\{1,2, \ldots, N\}$. Let $\left\{T_{n}\right\}_{n=1}^{\infty}$ be a sequence of nonexpansive self-mappings on $H$ and $\left\{\lambda_{n}\right\}$ be a sequence in $(0, b]$ for some $b \in(0,1)$. Let $F: H \rightarrow$ $H$ be a $\kappa$-Lipschitzian and $\eta$-strongly monotone operator with positive constants $\kappa, \eta>0$. Let $0<\mu<\left(2 \eta / \kappa^{2}\right)$ and $\tau=$ $1-\sqrt{1-\mu\left(2 \eta-\mu \kappa^{2}\right)}$. Let $f: H \rightarrow H$ be an l-Lipschitzian mapping with $0 \leq \gamma l<\tau$. Let $W_{n}$ be the $W$-mapping defined by (15) and $V$ be a $\bar{\gamma}$-strongly positive bounded linear operator with $\gamma l<(1+\mu) \bar{\gamma}$. Assume that $\Omega:=\bigcap_{n=1}^{\infty} \operatorname{Fix}\left(T_{n}\right) \cap \bigcap_{i=1}^{N} I\left(B_{i}, R_{i}\right)$ is nonempty. Let $\left\{\alpha_{n}\right\},\left\{\beta_{n}\right\}$, and $\left\{\sigma_{n}\right\}$ be three sequences in $(0,1)$. Assume that,

(i) $\lim _{n \rightarrow \infty} \sigma_{n}=\lim _{n \rightarrow \infty}\left(\alpha_{n} / \sigma_{n}\right)=0$, limsup $\sup _{n \rightarrow \infty}$ $\left(\alpha_{n+1} / \alpha_{n}\right)<\infty, \sum_{n=1}^{\infty} \sigma_{n}=\infty$ and

$$
0<\liminf _{n \rightarrow \infty} \beta_{n} \leq \limsup _{n \rightarrow \infty} \beta_{n}<1
$$

(ii) $\mu_{i} \in\left(0,2 \eta_{i}\right), i \in\{1,2, \ldots, N\}$;

(iii) $\sum_{n=1}^{\infty}\left(\left|\beta_{n+1}-\beta_{n}\right|+\left|\sigma_{n+1}-\sigma_{n}\right|\right)<\infty$. 
For a given arbitrary $x_{1} \in H$, let the sequence $\left\{x_{n}\right\}$ be generated iteratively by

$$
\begin{aligned}
z_{n}=J_{R_{N}, \mu_{N}}\left(I-\mu_{N} B_{N}\right) J_{R_{N-1}, \mu_{N-1}}\left(I-\mu_{N-1} B_{N-1}\right) & \\
& \cdots J_{R_{1}, \mu_{1}}\left(I-\mu_{1} B_{1}\right) x_{n} \\
y_{n}= & \alpha_{n}\left(u+\gamma f\left(x_{n}\right)\right)+\beta_{n} x_{n} \\
& +\left(\left(1-\beta_{n}\right) I-\alpha_{n}(I+\mu V)\right) W_{n} z_{n}, \\
x_{n+1}= & \sigma_{n} \gamma f\left(y_{n}\right)+\left(I-\sigma_{n} \mu F\right) W_{n} y_{n}, \quad \forall n \geq 1 .
\end{aligned}
$$

Then the sequence $\left\{x_{n}\right\}$ converges strongly to $x^{*} \in \Omega$, where $x^{*}=P_{\Omega}(I-(\mu F-\gamma f)) x^{*}$ is a unique solution of the VIP:

$$
\left\langle(\gamma f-\mu F) x^{*}, y-x^{*}\right\rangle \leq 0, \quad \forall y \in \Omega .
$$

Proof. Put $\Theta(x, y)=0, \varphi(x)=0$ for all $x, y \in C, A x=0$ for all $x \in H$ and $r_{n}=1$. Take $K(x)=(1 / 2)\|x\|^{2}$ for all $x \in$ $H$. Then we get $u_{n}=x_{n}$ in Theorem 20 and the conclusion follows.

\section{Conflict of Interests}

The authors declare that there is no conflict of interests regarding the publication of this paper.

\section{Acknowledgments}

This research was partially supported by the National Science Foundation of China (11071169), Innovation Program of Shanghai Municipal Education Commission (09ZZ133), and Ph.D. Program Foundation of Ministry of Education of China (20123127110002). This work was supported partly by the National Science Council of the Republic of China.

\section{References}

[1] J. L. Lions, Quelques Méthodes de Résolution des Problèmes aux Limites Non Lineaires, Dunod, Paris, France, 1969.

[2] R. Glowinski, Numerical Methods for Nonlinear Variational Problems, Springer, New York, NY, USA, 1984.

[3] W. Takahashi, Nonlinear Functional Analysis, Yokohama Publishers, Yokohama, Japan, 2000.

[4] J. T. Oden, Quantitative Methods on Nonlinear Mechanics, Prentice Hall, Upper Saddle River, NJ, USA, 1986.

[5] E. Zeidler, Nonlinear Functional Analysis and Its Applications, Springer, New York, NY, USA, 1985.

[6] G. M. Korpelevič, "An extragradient method for finding saddle points and for other problems," Ekonomika i Matematicheskie Metody, vol. 12, no. 4, pp. 747-756, 1976.

[7] A. J. Zaslavski, "The extragradient method for finding a common solution of a finite family of variational inequalities and a finite family of fixed point problems in the presence of computational errors," Journal of Mathematical Analysis and Applications, vol. 400, no. 2, pp. 651-663, 2013.

[8] A. Bnouhachem, M. Aslam Noor, and Z. Hao, "Some new extragradient iterative methods for variational inequalities," Nonlinear Analysis: Theory, Methods \& Applications, vol. 70, no. 3, pp. 1321-1329, 2009.
[9] I. Inchan, "Hybrid extragradient method for general equilibrium problems and fixed point problems in Hilbert space," Nonlinear Analysis: Hybrid Systems, vol. 5, no. 3, pp. 467-478, 2011.

[10] L.-C. Ceng and J.-C. Yao, "An extragradient-like approximation method for variational inequality problems and fixed point problems," Applied Mathematics and Computation, vol. 190, no. 1, pp. 205-215, 2007.

[11] N. Nadezhkina and W. Takahashi, "Weak convergence theorem by an extragradient method for nonexpansive mappings and monotone mappings," Journal of Optimization Theory and Applications, vol. 128, no. 1, pp. 191-201, 2006.

[12] N. Nadezhkina and W. Takahashi, "Strong convergence theorem by a hybrid method for nonexpansive mappings and Lipschitz-continuous monotone mappings," SIAM Journal on Optimization, vol. 16, no. 4, pp. 1230-1241, 2006.

[13] G. Cai and S. Bu, "Strong and weak convergence theorems for general mixed equilibrium problems and variational inequality problems and fixed point problems in Hilbert spaces," Journal of Computational and Applied Mathematics, vol. 247, pp. 34-52, 2013.

[14] J.-W. Peng and J.-C. Yao, "A new hybrid-extragradient method for generalized mixed equilibrium problems, fixed point problems and variational inequality problems," Taiwanese Journal of Mathematics, vol. 12, no. 6, pp. 1401-1432, 2008.

[15] L.-C. Ceng and J.-C. Yao, "A relaxed extragradient-like method for a generalized mixed equilibrium problem, a general system of generalized equilibria and a fixed point problem," Nonlinear Analysis: Theory, Methods \& Applications, vol. 72, no. 3-4, pp. 1922-1937, 2010.

[16] L.-C. Ceng, Q. H. Ansari, and S. Schaible, "Hybrid extragradient-like methods for generalized mixed equilibrium problems, systems of generalized equilibrium problems and optimization problems," Journal of Global Optimization, vol. 53, no. 1, pp. 6996, 2012.

[17] G. Cai and S. Bu, "Strong convergence theorems based on a new modified extragradient method for variational inequality problems and fixed point problems in Banach spaces," Computers \& Mathematics with Applications, vol. 62, no. 6, pp. 2567-2579, 2011.

[18] B.-S. He, Z.-H. Yang, and X.-M. Yuan, "An approximate proximal-extragradient type method for monotone variational inequalities," Journal of Mathematical Analysis and Applications, vol. 300, no. 2, pp. 362-374, 2004.

[19] W. Kumam and P. Kumam, "Hybrid iterative scheme by a relaxed extragradient method for solutions of equilibrium problems and a general system of variational inequalities with application to optimization," Nonlinear Analysis: Hybrid Systems, vol. 3, no. 4, pp. 640-656, 2009.

[20] G. Cai and S. Q. Bu, "Convergence analysis for variational inequality problems and fixed point problems in 2-uniformly smooth and uniformly convex Banach spaces," Mathematical and Computer Modelling, vol. 55, no. 3-4, pp. 538-546, 2012.

[21] L.-C. Ceng, Q. H. Ansari, M. M. Wong, and J.-C. Yao, "Mann type hybrid extragradient method for variational inequalities, variational inclusions and fixed point problems," Fixed Point Theory, vol. 13, no. 2, pp. 403-422, 2012.

[22] A. Bnouhachem, M. H. Xu, X.-L. Fu, and S. Zhaohan, "Modified extragradient methods for solving variational inequalities," Computers \& Mathematics with Applications, vol. 57, no. 2, pp. 230-239, 2009. 
[23] Y. Yao, Y.-C. Liou, and S. M. Kang, "Approach to common elements of variational inequality problems and fixed point problems via a relaxed extragradient method," Computers \& Mathematics with Applications, vol. 59, no. 11, pp. 3472-3480, 2010.

[24] G. Cai and S. Bu, "Hybrid algorithm for generalized mixed equilibrium problems and variational inequality problems and fixed point problems," Computers \& Mathematics with Applications, vol. 62, no. 12, pp. 4772-4782, 2011.

[25] Y. Yao, Y.-C. Liou, and J.-C. Yao, "New relaxed hybridextragradient method for fixed point problems, a general system of variational inequality problems and generalized mixed equilibrium problems," Optimization, vol. 60, no. 3, pp. 395-412, 2011.

[26] Y. Yao, Y. J. Cho, and Y.-C. Liou, "Algorithms of common solutions for variational inclusions, mixed equilibrium problems and fixed point problems," European Journal of Operational Research, vol. 212, no. 2, pp. 242-250, 2011.

[27] P. Sunthrayuth and P. Kumam, "Viscosity approximation methods based on generalized contraction mappings for a countable family of strict pseudo-contractions, a general system of variational inequalities and a generalized mixed equilibrium problem in Banach spaces," Mathematical and Computer Modelling, vol. 58, no. 11-12, pp. 1814-1828, 2013.

[28] K. R. Kazmi and S. H. Rizvi, "Iterative algorithms for generalized mixed equilibrium problems," Journal of the Egyptian Mathematical Society, vol. 21, no. 3, pp. 340-345, 2013.

[29] S. Takahashi and W. Takahashi, "Strong convergence theorem for a generalized equilibrium problem and a nonexpansive mapping in a Hilbert space," Nonlinear Analysis: Theory, Methods \& Applications, vol. 69, no. 3, pp. 1025-1033, 2008.

[30] Y. Yao, M. A. Noor, S. Zainab, and Y.-C. Liou, "Mixed equilibrium problems and optimization problems," Journal of Mathematical Analysis and Applications, vol. 354, no. 1, pp. 319329, 2009.

[31] L.-C. Ceng and J.-C. Yao, "A hybrid iterative scheme for mixed equilibrium problems and fixed point problems," Journal of Computational and Applied Mathematics, vol. 214, no. 1, pp. 186201, 2008.

[32] V. Colao, G. Marino, and H.-K. Xu, "An iterative method for finding common solutions of equilibrium and fixed point problems," Journal of Mathematical Analysis and Applications, vol. 344, no. 1, pp. 340-352, 2008.

[33] L. C. Ceng, A. Petruşel, and J. C. Yao, "Iterative approaches to solving equilibrium problems and fixed point problems of infinitely many nonexpansive mappings," Journal of Optimization Theory and Applications, vol. 143, no. 1, pp. 37-58, 2009.

[34] R. T. Rockafellar, "Monotone operators and the proximal point algorithm," SIAM Journal on Control and Optimization, vol. 14, no. 5, pp. 877-898, 1976.

[35] N.-J. Huang, "A new completely general class of variational inclusions with noncompact valued mappings," Computers \& Mathematics with Applications, vol. 35, no. 10, pp. 9-14, 1998.

[36] L.-C. Zeng, S.-M. Guu, and J.-C. Yao, "Characterization of $H$-monotone operators with applications to variational inclusions," Computers \& Mathematics with Applications, vol. 50, no. 3-4, pp. 329-337, 2005.

[37] Y.-P. Fang, N.-J. Huang, and H. B. Thompson, "A new system of variational inclusions with $(H, \eta)$-monotone operators in Hilbert spaces," Computers \& Mathematics with Applications, vol. 49, no. 2-3, pp. 365-374, 2005.
[38] S.-S. Zhang, J. H. W. Lee, and C. K. Chan, "Algorithms of common solutions to quasi variational inclusion and fixed point problems," Applied Mathematics and Mechanics (English Edition), vol. 29, no. 5, pp. 571-581, 2008.

[39] J. G. O'Hara, P. Pillay, and H.-K. Xu, "Iterative approaches to convex feasibility problems in Banach spaces," Nonlinear Analysis: Theory, Methods \& Applications, vol. 64, no. 9, pp. 2022-2042, 2006.

[40] L.-C. Ceng and J.-C. Yao, "Hybrid viscosity approximation schemes for equilibrium problems and fixed point problems of infinitely many nonexpansive mappings," Applied Mathematics and Computation, vol. 198, no. 2, pp. 729-741, 2008.

[41] K. Goebel and W. A. Kirk, Topics on Metric Fixed-Point Theory,, Cambridge University Press, Cambridge, UK, 1990.

[42] H. K. Xu and T. H. Kim, "Convergence of hybrid steepestdescent methods for variational inequalities," Journal of Optimization Theory and Applications, vol. 119, no. 1, pp. 185-201, 2003.

[43] H.-K. Xu, "Iterative algorithms for nonlinear operators," Journal of the London Mathematical Society. Second Series, vol. 66, no. 1, pp. 240-256, 2002.

[44] G. Marino and H.-K. Xu, "Weak and strong convergence theorems for strict pseudo-contractions in Hilbert spaces," Journal of Mathematical Analysis and Applications, vol. 329, no. 1, pp. 336-346, 2007. 


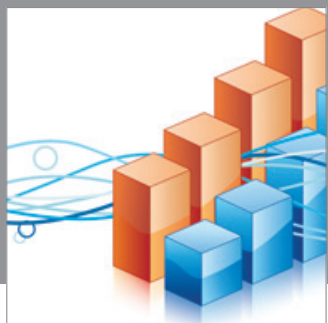

Advances in

Operations Research

mansans

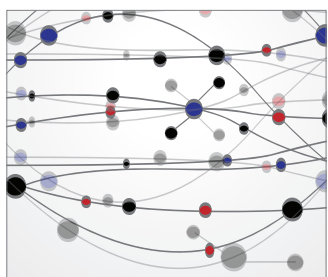

The Scientific World Journal
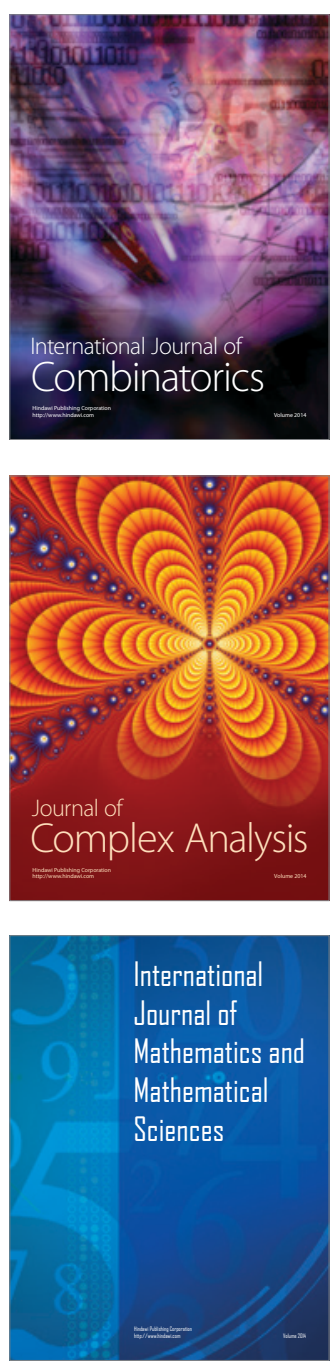
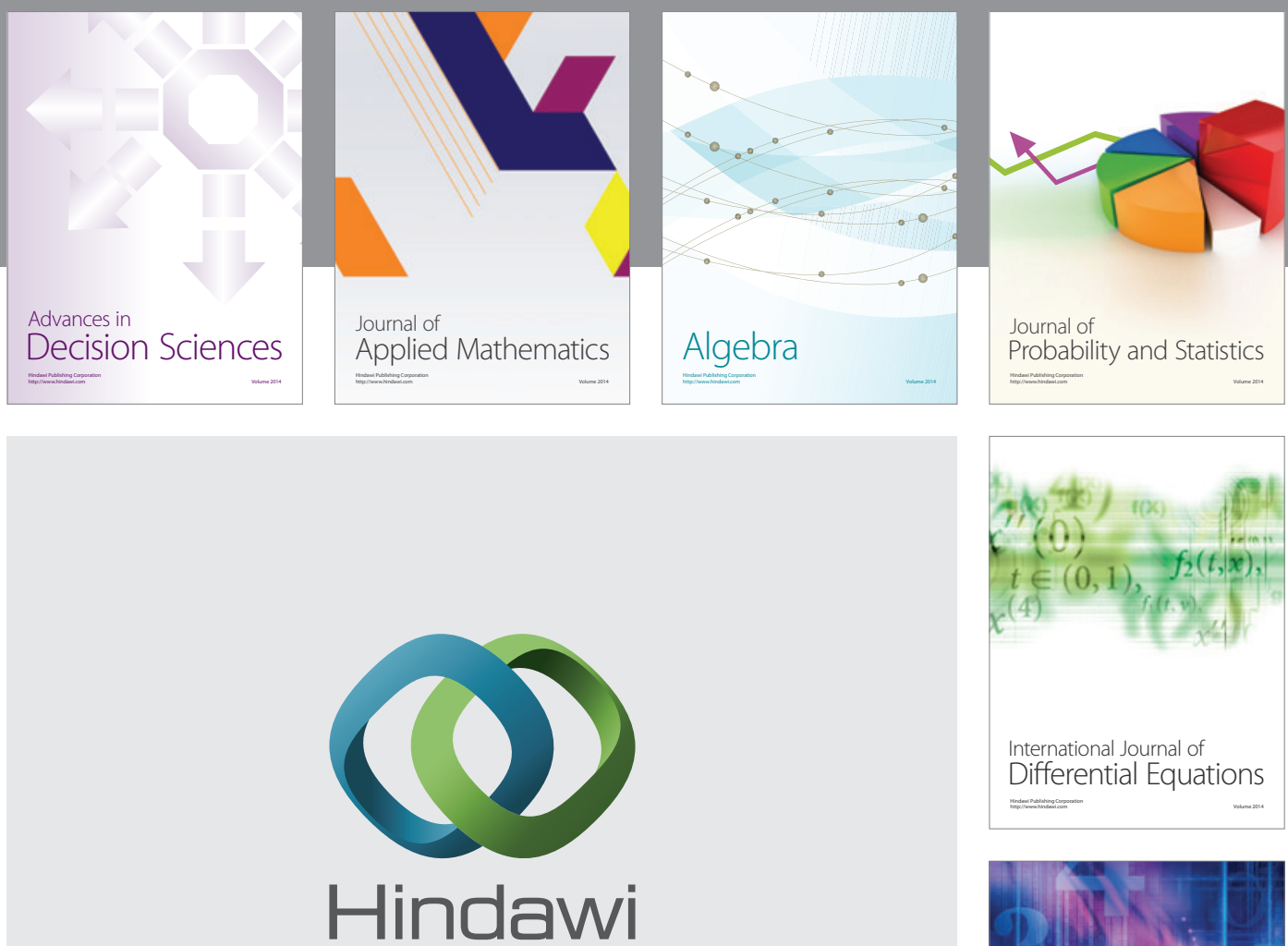

Submit your manuscripts at http://www.hindawi.com
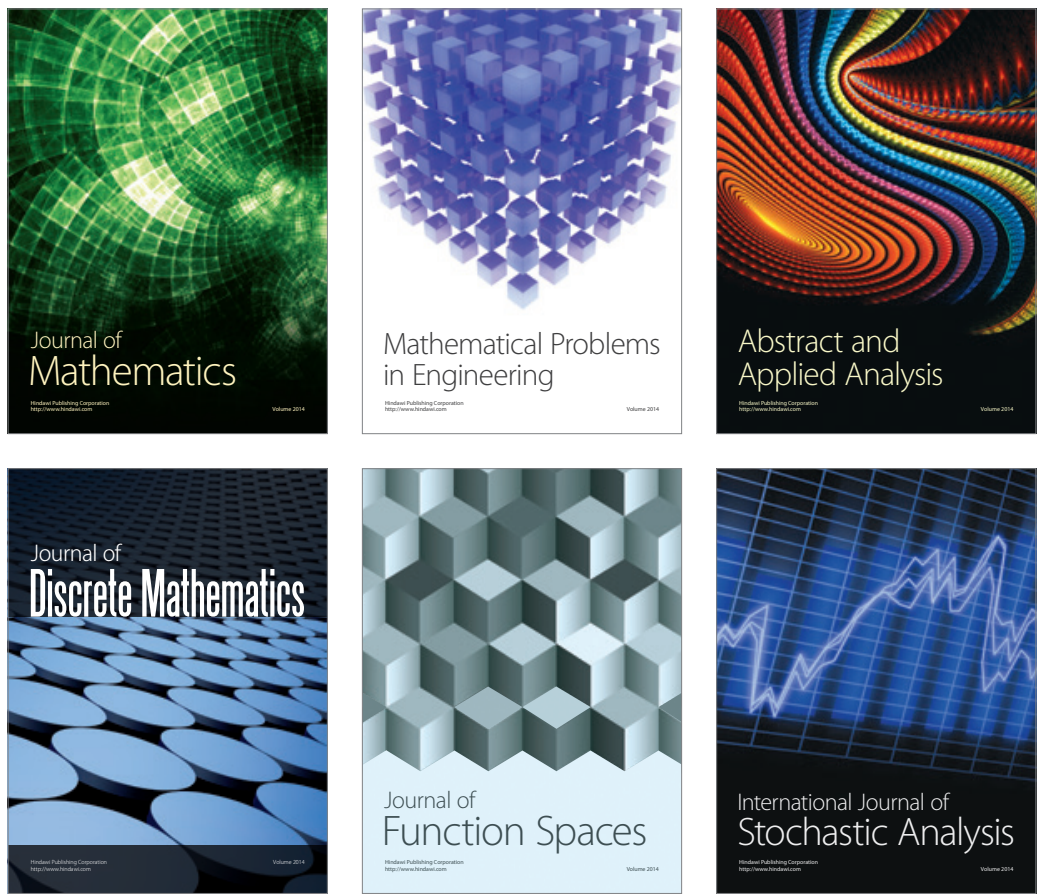

Journal of

Function Spaces

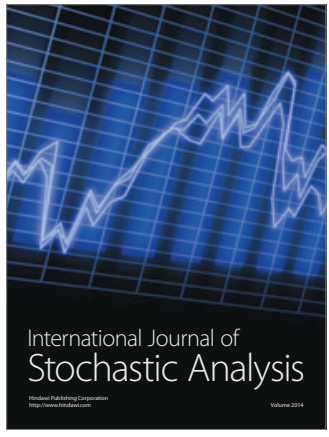

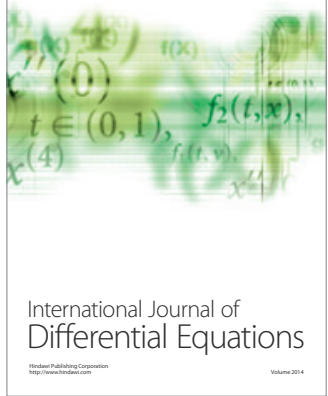
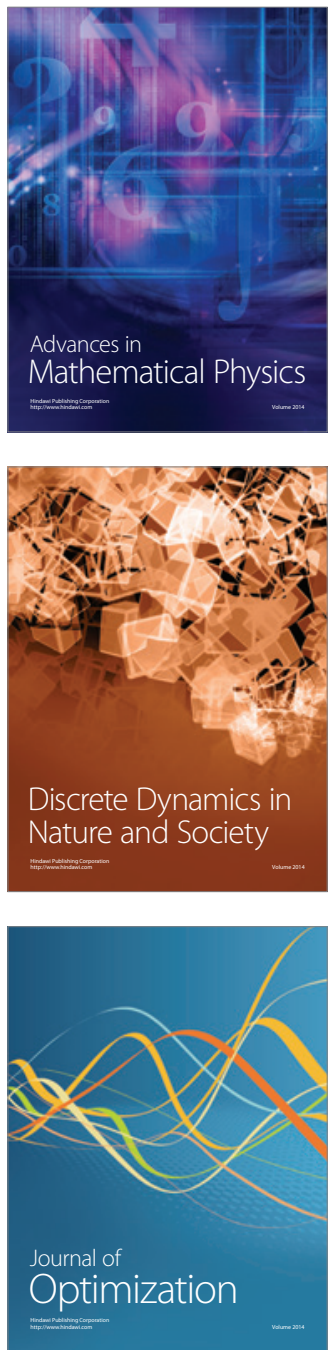